BMJ Open Diabetes Research \& Care

\section{Long-term safety and efficacy of alogliptin, a DPP-4 inhibitor, in patients with type 2 diabetes: a 3-year prospective, controlled, observational study (J-BRAND Registry)}

Kohjiro Ueki (D) , ${ }^{1}$ Yukio Tanizawa, ${ }^{2}$ Jiro Nakamura, ${ }^{3}$ Yuichiro Yamada, ${ }^{4}$ Nobuya Inagaki (i) ${ }^{5}$ Hirotaka Watada, ${ }^{6}$ lichiro Shimomura, ${ }^{7}$ Rimei Nishimura, ${ }^{8}$ Hideaki Miyoshi (1) , ${ }^{9}$ Atsuko Abiko, ${ }^{10}$ Hideki Katagiri, ${ }^{11}$ Michio Hayashi, ${ }^{12}$ Akira Shimada (D) , ${ }^{13}$ Keiko Naruse, ${ }^{14}$ Shimpei Fujimoto, ${ }^{15}$ Masazumi Fujiwara, ${ }^{16}$ Kenichi Shikata, ${ }^{17}$ Yosuke Okada, ${ }^{18}$ Eiichi Araki, ${ }^{19}$ Tsutomu Yamazaki, ${ }^{20}$ Takashi Kadowaki (D) ,21,22 J-BRAND Registry Group
To cite: Ueki K, Tanizawa Y, Nakamura J, et al. Longterm safety and efficacy of alogliptin, a DPP-4 inhibitor, in patients with type 2 diabetes: a 3-year prospective, controlled, observational study (J-BRAND Registry). BMJ Open Diab Res Care 2021;9:e001787. doi:10.1136/ bmjdrc-2020-001787

- Additional material is published online only. To view please visit the journal online (http://dx.doi.org/10.1136/ bmjdrc-2020-001787).

Received 23 July 2020 Revised 15 November 2020 Accepted 5 December 2020

Check for updates

(c) Author(s) (or their employer(s)) 2021. Re-use permitted under CC BY-NC. No commercial re-use. See rights and permissions. Published by BMJ.

For numbered affiliations see end of article.

Correspondence to Dr Takashi Kadowaki; t-kadowaki@toranomon.kkr. or.jp

\section{ABSTRACT}

Introduction Given an increasing use of dipeptidyl peptidase-4 (DPP-4) inhibitors to treat patients with type 2 diabetes mellitus in the real-world setting, we conducted a prospective observational study (Japan-based Clinical Research Network for Diabetes Registry: J-BRAND Registry) to elucidate the safety and efficacy profile of long-term usage of alogliptin.

Research design and methods We registered 5969 patients from April 2012 through September 2014, who started receiving alogliptin (group A) or other classes of oral hypoglycemic agents (OHAs; group B), and were followed for 3 years at 239 sites nationwide. Safety was the primary outcome. Symptomatic hypoglycemia, pancreatitis, skin disorders of non-extrinsic origin, severe infections, and cancer were collected as major adverse events (AEs). Efficacy assessment was the secondary outcome and included changes in hemoglobin A1c (HbA1c), fasting blood glucose, fasting insulin and urinary albumin. Results Of the registered, 5150 (group A: 3395 and group B: 1755) and 5096 (3358 and 1738) were included for safety and efficacy analysis, respectively. Group A patients mostly (>90\%) continued to use alogliptin. In group $B$, biguanides were the primary agents, while DPP-4 inhibitors were added in up to $~ 36 \%$ of patients. The overall incidence of AEs was similar between the two groups (42.7\% vs $42.2 \%)$. Kaplan-Meier analysis revealed the incidence of cancer was significantly higher in group $A$ than in group $B(7.4 \%$ vs $4.8 \%, p=0.040)$, while no significant incidence difference was observed in the individual cancer. Multivariate Cox regression analysis revealed that the imbalanced patient distribution (more elderly patients in group A than in group B), but not alogliptin usage per se, contributed to cancer development. The incidence of other major AE categories was with no between-group difference. Between-group difference was not detected, either, in the incidence of microvascular and macrovascular complications. HbA1c and fasting glucose decreased significantly at the 0.5 -year visit and nearly plateaued thereafter in both groups.

Conclusions Alogliptin as a representative of DPP-4 inhibitors was safe and durably efficacious when used

\section{Significance of this study}

What is already known about this subject?

- Safety profile was proposed for dipeptidyl peptidase-4 (DPP-4) inhibitors in the previous studies, but the evidence was generally limited to cardiovascular events, hypoglycemia, pancreatitis, and pancreatic cancer, obtained through relatively short-term observations in patients with type 2 diabetes with prior cardiovascular history.

- Some of the studies raised a concern about the increased risk of heart failure with DPP-4 inhibitors.

What are the new findings?

- Alogliptin, as a representative of DPP-4 inhibitors, was safe and efficacious for a 3-year period.

- The results strongly suggest the safe and durably efficacious profile of DPP-4 inhibitors in comparison with other oral hypoglycemic agents including biguanides.

How might these results change the focus of research or clinical practice?

- DPP-4 inhibitors can be more recommended for glycemic control in elderly patients with type 2 diabetes mellitus.

- Bullous pemphigoid, a possible risk suggested in association with the use of DPP-4 inhibitors, should be further monitored in clinical practice.

alone or with other OHAs for patients with type 2 diabetes in the real world setting.

\section{INTRODUCTION}

Type 2 diabetes mellitus is a pandemic that threatens health and economy worldwide because of its various complications. ${ }^{1-3}$ Different classes of agents with different modes of action have become available to 
treat the disease, such as biguanides, thiazolidinediones, sulfonylureas, glinides, $\alpha$-glucosidase inhibitors, and insulin therapy, and more recently, incretins and related compounds including glucagon-like peptide-1 (GLP-1) receptor agonists and dipeptidyl peptidase-4 (DPP-4) inhibitors, and sodium-glucose cotransporter2 (SGLT2) inhibitors. ${ }^{4}$ Among those, DPP-4 inhibitors have been of clinical attention in recent years because of the proposed low risk of hypoglycemic events and weight gain. ${ }^{56}$

Several large-scale clinical trials were conducted using DPP-4 inhibitors, such as Saxagliptin Assessment of Vascular Outcomes Recorded in patients with diabetes mellitus-Thrombolysis in Myocardial Infarction (SAVORTIMI) 53 for saxagliptin, ${ }^{7}$ Examination of Cardiovascular Outcomes: Alogliptin vs Standard of Care (EXAMINE) for alogliptin, ${ }^{8}$ Trial Evaluating Cardiovascular Outcomes with Sitagliptin (TECOS) for sitagliptin, ${ }^{9}$ and Cardiovascular and Renal Microvascular Outcome Study with Linagliptin (CARMELINA) for linagliptin, ${ }^{10}$ but were to mainly evaluate the safety (particularly on cardiovascular events) and efficacy of the individual drugs. While these trials showed the safe profile of DPP-4 inhibitors in terms of the risk of cardiovascular disease ${ }^{11}$ as well as hypoglycemia, pancreatitis, and pancreatic cancer, the study periods were generally short and most of the participants had prior history of cardiovascular disease. Moreover, SAVOR-TIMI53 and EXAMINE raised a concern about the increased risk of heart failure with the drug class, ${ }^{12}$ especially saxagliptin, ${ }^{7}$ and alogliptin to a lesser extent. ${ }^{813}$ It is thus important to examine DPP-4 inhibitors for a longer period in the subjects who are not at high cardiovascular risk to entirely clarify the safety issues suggested and unidentified for the drug class. Registry studies must be useful for this purpose, and indeed several reports using registries have shown the safety and efficacy of the class as real-world evidence. ${ }^{514}{ }^{15}$ It should be noted, however, that these studies were retrospective ${ }^{1314}$ or non-controlled, ${ }^{5}$ or used the short-term claim databases, and lack various important information such as anthropometric and laboratory data. ${ }^{14}$

To more precisely evaluate the safety and efficacy of DPP-4 inhibitors, we conducted a 3-year, large-scale, prospective, controlled, observational study (Japanbased Clinical Research Network for Diabetes Registry: J-BRAND Registry) in the Japanese patients with type 2 diabetes. The study was designed as a concurrently controlled one: patients started the study with initiation of alogliptin (brand name: Nesina) as a representative of DPP-4 inhibitors (group A), while other patients started with initiation of other classes of oral hypoglycemic agents (OHAs) for comparison (group B, see Research design and methods section). ${ }^{16}$ The relatively long-term, non-intervening (ie, real world) design of J-BRAND Registry was expected to surpass the limitations associated with the aforementioned, conventional cohort studies. Furthermore, the study allowed the investigators to follow up any safety events including macrovascular as well as microvascular events occurring following the usage of DPP-4 inhibitors and other OHAs. We report here the safety and efficacy profile of alogliptin in the real-world setting.

\section{RESEARCH DESIGN AND METHODS}

\section{Study treatment and procedures}

The overall study procedures were already described ${ }^{16}$ in line with the principles of the Declaration of Helsinki and the Harmonised Tripartite Guideline for Good Clinical Practice from the International Council for Harmonisation, and approved centrally by MINS IRB (Tokyo, Japan) and then by the Institutional Review Board set up at each institutional organization. Patients aged 20 years or older with diagnosed type 2 diabetes participated in this study (see box 1 for the detailed patient criteria in our previous article). ${ }^{16}$ They provided written informed consent at the time of study registration. Patients were separated into two predefined groups, where they initiated the study with either alogliptin (group A) or non-DPP-4 inhibitor OHAs (group B), respectively, with or without concomitant use of different classes of OHAs primarily depending on their condition. The patients of each group were further sub-grouped according to the type of treatment initiation as "start", "addition", or "switch", where alogliptin or non-DPP-4 inhibitor OHA was newly started, added to the previous treatment, or switched from the previous $\mathrm{OHA}(\mathrm{s})$ at the time of or within 3 months prior to the study registration (see figure 2 in our previous article). ${ }^{16}$ Treatment with $\mathrm{OHA}(\mathrm{s})$ was provided in daily clinical practice and was allowed to change or discontinue as per the package insert for each OHA. ${ }^{17}$ For example, Nesina as a representative of DPP-4 inhibitors was administered at a dose of $25 \mathrm{mg}$ once daily, while either 6.25 or $12.5 \mathrm{mg}$ daily was used at physician's discretion in the patients associated with moderate-to-severe kidney malfunction. Non-OHA antidiabetic therapies and/or treatments for concurrent medical conditions were also provided when needed. The patients were to visit their sites for assessment every 6 months during the 3-year study period and the data were registered via a customized electronic data capture system.

\section{Outcomes}

The primary outcome of the present study was all adverse events (AEs). The overall schedule and essential and optional items for observations were as in tables 1 and 2 of our previous article. ${ }^{16}$ Any AE was assessed with its term, seriousness, severity, causality to OHA(s) or other treatments used, date of onset, date of resolution, frequency, action taken on OHAs (and other treatments), and consequence. Symptomatic hypoglycemia, pancreatitis (acute or chronic), skin disorders of non-extrinsic origin, severe infections, and cancer were collected as major AEs. ${ }^{18-22}$ Microvascular and macrovascular complications were also collected. AE terms were referred to MedDRA V.15.1. The secondary outcome was efficacy of alogliptin and 
included the levels of hemoglobin A1c (HbA1c), fasting blood glucose, fasting insulin, and urinary albumin.

Other measurements (concurrent medical conditions, laboratory parameters, physical examinations, chest X-ray, and standard 12-lead ECG) were performed as described. $^{16}$

\section{Statistical analysis}

Three different patient populations, full analysis set (FAS), safety analysis set (SAS), and efficacy analysis set (EAS), were defined for statistical analysis in the present study. ${ }^{16}$ SAS was the primary set for the analysis of safety and microvascular/macrovascular complications, while efficacy was analyzed using EAS.

Cumulative incidence of the major AEs (symptomatic hypoglycemia, pancreatitis acute or chronic, skin disorders of non-extrinsic origin, severe infections, and cancer) and microvascular complications were analyzed by the Kaplan-Meier method and log-rank test for comparison between group A and group B. Cox regression analysis was performed as appropriate. Changes from baseline of HbAlc and other efficacy endpoints were compared between the groups by two-sample $\mathrm{t}$ test. All statistical analyses were performed using SAS V.9.4. Note the abbreviation "SAS" was exclusively used to denote "safety analysis set" in the text.

\section{RESULTS}

\section{Disposition and baseline characteristics of patients}

The study was conducted from April 1, 2012 to December 31,2017 . Although we initially planned to recruit 10,000 patients each in group A and group B ${ }^{16}$ a total of 5969 subjects were registered until September 30, 2014 at 239 institutional sites nationwide.

Figure 1 depicts a diagram of the analysis sets. Of 5969 registered, 5745 were with baseline measurements, and 5208 were included in the FAS population (3424 in group A and 1784 in group B) after 537 excluded mainly due to no drug newly administered for study initiation (344),

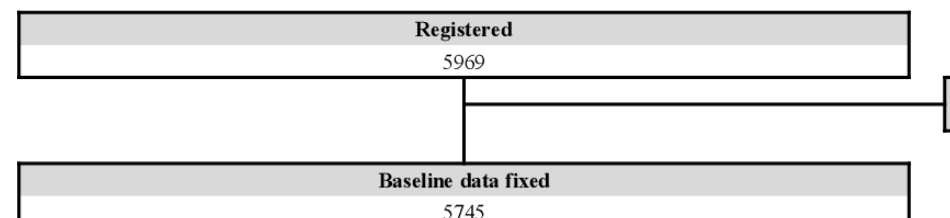

\begin{tabular}{|cccc|cccc|}
\hline \multicolumn{8}{|c|}{ Full Analysis Set (FAS) } \\
\hline \multicolumn{1}{|c|}{ Group A } & & \multicolumn{4}{c|}{ Group B } \\
\hline All & Start & Addition & Switch & All & Start & Addition & Switch \\
3424 & 809 & 1125 & 1490 & 1784 & 853 & 449 & 482 \\
\hline
\end{tabular}

Baseline data unfixed

\begin{tabular}{|l|l|}
\hline \multicolumn{1}{|c|}{ Baseline data unfixed } & 224 \\
\hline \multicolumn{1}{|c|}{ Excluded from FAS population } & 537 \\
\hline \multicolumn{2}{|c|}{} \\
\hline No drug newly administered for 'start', 'addition' or 'switch' & 344 \\
Lost to follow-up after Day 0 (= the time of 'start', 'addition' or 'switch') & 172 \\
Continuing use of DPP-4 inhibitor (alogliptin) from prior to Day 0 & 137 \\
Continuing use of DPP-4 inhibitors (except for alogliptin) from prior to Day 0 & 46 \\
Initiation of new drug (for 'start', 'addition' or 'switch') beyond the preset & 39 \\
allowance (Day0 + within 3 days) & 13 \\
Use of insulin formulation and/or GLP-1 receptor agonist at Day 0 & 10 \\
No drug newly administered for 'start', 'addition' or 'switch' until the day of & 39 \\
registration & 5 \\
Category of treatment initiation ('start', 'addition' or 'switch') unknown & 3 \\
Major protocol violation: informed consent provided prior to agreement & 2 \\
Major protocol violation: duplicate registration & 2 \\
Consent withdrawal at baseline & 2 \\
\hline Use of previous investigational drug (SYR-472= zafatek) &
\end{tabular}

\begin{tabular}{|lcc|}
\hline \multicolumn{1}{|c|}{ Excluded from SAS population } & Group A & Group B \\
\hline Discontinuation due to major protocol violation post-baseline & 29 & 29 \\
Violation of patient criteria at baseline & 3 & 20 \\
Other violation of patient criteria & 0 & 5 \\
Use of other investigational drug at Day 0 & 0 & 2 \\
\hline
\end{tabular}

\begin{tabular}{|cccc|cccc|}
\hline \multicolumn{8}{|c|}{ Safety Analysis Set (SAS) } \\
\hline Group A & & \multicolumn{4}{c|}{ Group B } \\
\hline All & Start & Addition & Switch & All & Start & Addition & Switch \\
3395 & 806 & 1118 & 1471 & 1755 & 844 & 445 & 466 \\
\hline
\end{tabular}


Table 1 Baseline characteristics of study patients

\begin{tabular}{|c|c|c|c|c|}
\hline & & Group A (N=3395) & Group B (N=1755) & P value \\
\hline \multirow[t]{2}{*}{ Sex, n (\%) } & Male & $2098(61.8)$ & $1074(61.2)$ & 0.675 \\
\hline & Female & $1297(38.2)$ & $681(38.8)$ & \\
\hline Age (years) & & $65.0(11.8)$ & $61.7(12.5)$ & $<0.001^{\star \star *}$ \\
\hline Duration of type 2 diabetes (years) & & $9.55(8.33)$ & $7.34(7.70)$ & $<0.001^{\star * *}$ \\
\hline \multirow[t]{3}{*}{ Smoking status, n (\%) } & No & $1752(51.6)$ & $855(48.7)$ & $<0.001^{\star \star \star}$ \\
\hline & Current & $616(18.1)$ & $402(22.9)$ & \\
\hline & Previous & $1027(30.3)$ & $498(28.4)$ & \\
\hline Height (cm) & & $161.1(9.3)$ & $161.8(9.2)$ & $0.012^{*}$ \\
\hline Weight (kg) & & $65.12(14.21)$ & $67.98(15.00)$ & $<0.001^{\star \star \star}$ \\
\hline $\mathrm{BMI}\left(\mathrm{kg} / \mathrm{m}^{2}\right)$ & & $24.99(4.45)$ & $25.85(4.62)$ & $<0.001^{* * *}$ \\
\hline $\mathrm{HbA1c}(\%) \ddagger$ & & $7.58(1.274)$ & $7.86(1.626)$ & $<0.001^{\star \star \star}$ \\
\hline Fasting blood glucose (mg/dL) & & $153.9(51.32)$ & $157.2(52.15)$ & 0.136 \\
\hline Fasting insulin $(\mu \mathrm{U} / \mathrm{mL})$ & & $9.33(9.63)$ & $10.46(11.71)$ & 0.140 \\
\hline Casual blood glucose (mg/dL) & & $175.7(68.71)$ & $186.9(75.62)$ & $<0.001^{\star * *}$ \\
\hline Systolic blood pressure $(\mathrm{mmHg})$ & & $131.3(16.90)$ & $133.0(18.37)$ & $0.009^{\star \star}$ \\
\hline Diastolic blood pressure $(\mathrm{mmHg})$ & & $74.6(11.50)$ & $76.8(12.81)$ & $<0.001^{\star * *}$ \\
\hline Pulse rate (bpm) & & $77.3(12.44)$ & $77.1(12.80)$ & 0.719 \\
\hline Total cholesterol (mg/dL) & & $187.9(34.94)$ & $195.6(39.50)$ & $<0.001^{\star * \star}$ \\
\hline HDL cholesterol (mg/dL) & & $54.8(17.70)$ & $54.1(21.70)$ & 0.369 \\
\hline LDL cholesterol (mg/dL) & & $109.2(31.11)$ & $115.5(33.84)$ & $<0.001^{\star \star \star}$ \\
\hline Fasting triglycerides (mg/dL) & & $136.7(84.12)$ & $143.7(92.88)$ & 0.060 \\
\hline Serum creatinine (mg/dL) & & $0.832(1.744)$ & $0.796(0.943)$ & 0.394 \\
\hline Urinary albumin (mg/g·Cre) & & $91.37(337.54)$ & $103.12(355.48)$ & 0.229 \\
\hline
\end{tabular}

Values are mean (SD) unless otherwise specified.

${ }^{*} p<0.05$, ${ }^{* *} p<0.01$ and $\geq 0.001$, and ${ }^{* * *} p<0.001$.

†Patients were compared between group A and group B for sex, and smoking status by $\chi^{2}$ test, for urinary albumin by Wilcoxon rank-sum test and for the other categories by t-test.

$\ddagger \%$ of mean $\mathrm{HbA} 1 \mathrm{c}$ was converted to $\mathrm{mmol} / \mathrm{mol}$ as 59 and 62 , respectively.

BMI, body mass index; HbA1c, hemoglobin A1c; HDL, high-density lipoprotein; LDL, low-density lipoprotein.

loss to follow-up after day 0 (172; day $0=$ the time of study initiation), and continuing use of alogliptin from prior to day 0 (137). Following 29 each excluded from the FAS population, 5150 (3395 and 1755) were included in the SAS population. Fifty-four (37 and 17) patients were further excluded due to loss to follow-up and then the EAS population included 5096 (3358 and 1738) patients. The statistical power was $96.6 \%$ for group A and $82.7 \%$ for group B to detect an AE occurring in SAS population at $0.1 \%$ incidence.

The percentage of study completers was comparable between the two groups of SAS population $(2374 / 3395=69.9 \%$ and $1239 / 1755=70.6 \%)$. During the study period, $887(26.1 \%)$ group A patients and 471 $(26.8 \%)$ group B patients discontinued the study mainly due to loss to follow-up (334 (9.8\%) and $211(12.0 \%)$ ) and voluntary withdrawal (191 (5.6\%) and $91(5.2 \%))$.

Baseline characteristics of the SAS population are summarized in table 1 . Mean duration of type 2 diabetes was significantly longer in group A patients than in group B patients $(9.55$ vs 7.34 years; $\mathrm{p}<0.001)$. Statistically significant between-group differences were also found in age $(65.0$ vs 61.7 years; $\mathrm{p}<0.001)$, smoking status ( $p<0.001)$, height $(161.1$ vs $161.8 \mathrm{~cm} ; \mathrm{p}=0.012)$, weight (65.12 vs $67.98 \mathrm{~kg} ; \mathrm{p}<0.001$ ), and body mass index (BMI; 24.99 vs $\left.25.85 \mathrm{~kg} / \mathrm{m}^{2} ; \mathrm{p}<0.001\right)$.

Mean values of HbAlc and casual blood glucose were significantly higher in group B than in group A (7.86 vs $7.58 \%$; 62 vs $59 \mathrm{mmol} / \mathrm{mol}, \mathrm{p}<0.001$ and 186.9 vs $175.7 \mathrm{mg} / \mathrm{dL}, \mathrm{p}<0.001)$. Other efficacy-linked parameters (fasting blood glucose, fasting serum insulin, and urinary albumin) were comparable between the two groups. Systolic and diastolic blood pressures were higher in group B patients $(133.0$ vs $131.3 \mathrm{mmHg}, \mathrm{p}=0.009$; and 76.8 vs $74.6 \mathrm{mmHg}, \mathrm{p}<0.001)$. Total cholesterol and lowdensity lipoprotein (LDL) cholesterol were also higher in group B (195.6 vs $187.9 \mathrm{mg} / \mathrm{dL}, \mathrm{p}<0.001$ and 115.5 vs $109.2 \mathrm{mg} / \mathrm{dL}, \mathrm{p}<0.001$, respectively). Other baseline laboratory and vital parameters are listed in online supplemental table 1 and were either with no between-group difference or were deemed clinically less significant even if with statistical difference. 
Table 2 Usage of oral hypoglycemic agents

\begin{tabular}{|c|c|c|c|}
\hline \multirow[b]{2}{*}{ Group } & \multirow[b]{2}{*}{ Drug class } & \multicolumn{2}{|l|}{ Visit (year) } \\
\hline & & Baseline & 3.0 \\
\hline \multirow[t]{9}{*}{ A } & Patients & 3395 (100.0\%) & $1839(100.0 \%)$ \\
\hline & No use of oral hypoglycemic drugs & $0(0.0 \%)$ & $48(2.6 \%)$ \\
\hline & Sulfonylureas & $1160(34.2 \%)$ & $619(33.7 \%)$ \\
\hline & Rapid-acting insulin secretagogues & $100(2.9 \%)$ & $128(7.0 \%)$ \\
\hline & $\alpha$-Glucosidase inhibitors & $539(15.9 \%)$ & $321(17.5 \%)$ \\
\hline & Biguanides & $1352(39.8 \%)$ & $894(48.6 \%)$ \\
\hline & Thiazolidinediones & $508(15.0 \%)$ & $325(17.7 \%)$ \\
\hline & DPP-4 inhibitors & 3395 (100.0\%) & $1687(91.7 \%)$ \\
\hline & SGLT2 inhibitors & $1(0.03 \%)$ & $160(8.7 \%)$ \\
\hline \multirow[t]{9}{*}{$\mathrm{B}$} & Patients & $1755(100.0 \%)$ & $965(100.0 \%)$ \\
\hline & No use of oral hypoglycemic drugs & $0(0.0 \%)$ & $35(3.6 \%)$ \\
\hline & Sulfonylureas & $505(28.8 \%)$ & $235(24.4 \%)$ \\
\hline & Rapid-acting insulin secretagogues & $347(19.8 \%)$ & $185(19.2 \%)$ \\
\hline & $\alpha$-Glucosidase inhibitors & $435(24.8 \%)$ & $215(22.3 \%)$ \\
\hline & Biguanides & $1192(67.9 \%)$ & $622(64.5 \%)$ \\
\hline & Thiazolidinediones & $342(19.5 \%)$ & $157(16.3 \%)$ \\
\hline & DPP-4 inhibitors & $0(0.0 \%)$ & $352(36.5 \%)$ \\
\hline & SGLT2 inhibitors & $21(1.2 \%)$ & $108(11.2 \%)$ \\
\hline
\end{tabular}

DPP, dipeptidyl peptidase; SGLT, sodium-glucose cotransporter.

There was a notable difference in OHA usage between the two groups. Before study registration, group A patients used more OHAs compared with group B patients (mean number of OHAs: 1.5 vs 0.8 ). Nearly twice more patients used two or more OHAs in group A $(45.5 \%$ vs $23.0 \%)$, and a comparable percentage $(30.7 \%$ vs $28.9 \%)$ was with oral monotherapies while a lower percentage $(23.7 \%$ vs $48.1 \%$ ) was with no use of OHAs. This tendency was also observed at baseline as 2.1 versus 1.6, while a gradual increase in group B patients as 2.3 versus 2.0 at the 3-year study end. The time-dependent changes of the usage of different OHA classes are profiled in table 2 and online supplemental figure 1. All group A patients received a DPP-4 inhibitor (alogliptin) at baseline as defined in the study protocol. ${ }^{16}$ While the real-world setting allowed therapeutic changes with different drug classes, group A patients mostly $(>90 \%)$ continued to use alogliptin (or other DPP-4 inhibitors) throughout the study. Biguanides and sulfonylureas were the two secondary dominants received by the group A patients, while the group B patients used biguanides as the primary agent at baseline as expected in the current clinical practice. It was interesting that the use of DPP-4 inhibitors gradually increased (up to $\sim 36 \%$ ) in group B through the study progress and became the second dominant over sulfonylureas at the later stage. Non-OHA therapies (insulin formulations and GLP-1 receptor agonists) were also used in an increasing number of patients in both groups up to ca. $4.2 \%$ and $2.4 \%$ in group A and $2.7 \%$ and $2.0 \%$ in group $\mathrm{B}$, respectively.

\section{Primary outcome}

AEs were collected as the primary outcome in this study, and all reported AEs are tabulated in online supplemental table 2. The overall incidence of AEs was similar between group A and group B (42.7\% vs $42.2 \%$; $\mathrm{p}=0.744)$. The

Table 3 Cumulative incidence of major adverse events

\begin{tabular}{|c|c|c|c|}
\hline & $\begin{array}{l}\text { Group A } \\
(\mathrm{N}=3395)\end{array}$ & $\begin{array}{l}\text { Group B } \\
(\mathrm{N}=1755)\end{array}$ & $P$ value \\
\hline & n (\%) & n (\%) & Group A vs B \\
\hline $\begin{array}{l}\text { Symptomatic } \\
\text { hypoglycemia }\end{array}$ & 104 (3.9) & 45 (3.2) & 0.317 \\
\hline Pancreatitis acute & $5(0.2)$ & $3(0.9)$ & 0.861 \\
\hline Pancreatitis chronic & $2(0.1)$ & $0(0.0)$ & 0.310 \\
\hline $\begin{array}{l}\text { Skin disorders of } \\
\text { non-extrinsic origin }\end{array}$ & $201(7.9)$ & $90(6.2)$ & 0.240 \\
\hline Severe infections & $71(2.7)$ & $28(2.0)$ & 0.222 \\
\hline Cancer & $162(7.4)$ & $62(4.8)$ & $0.040^{\star}$ \\
\hline
\end{tabular}

Cumulative incidence (\%) of major AEs was calculated per observation period by the Kaplan-Meier method (see figure 2). $P$ values were by log-rank test. Potential factors contributing to cancer development were detailed by Cox regression analysis as shown in online supplemental table 3.

${ }^{*} p$ value with significance level smaller than 0.05 . 
A Symptomatic hypoglycemia

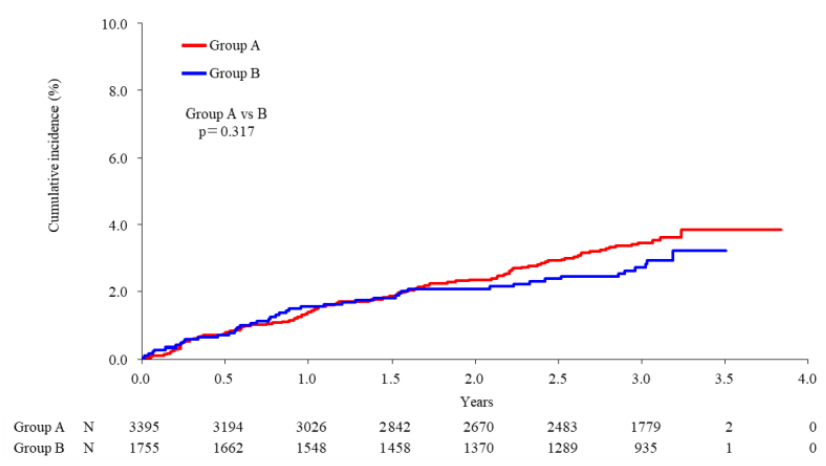

C Severe infections

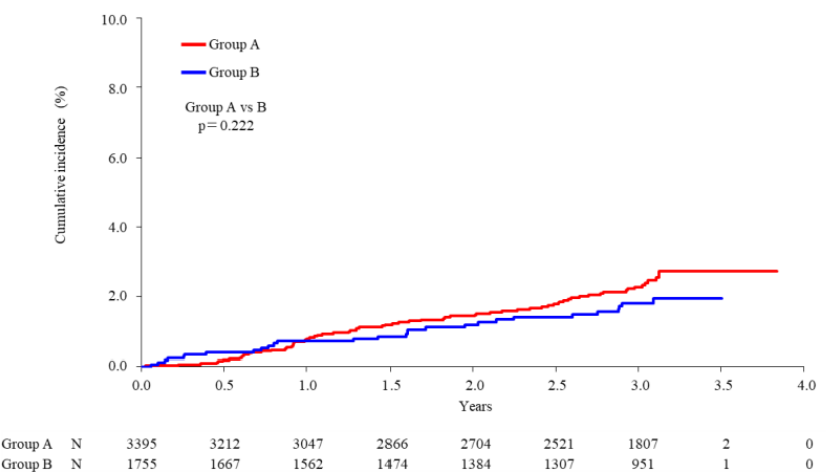

B Skin disorders of non-extrinsic origin

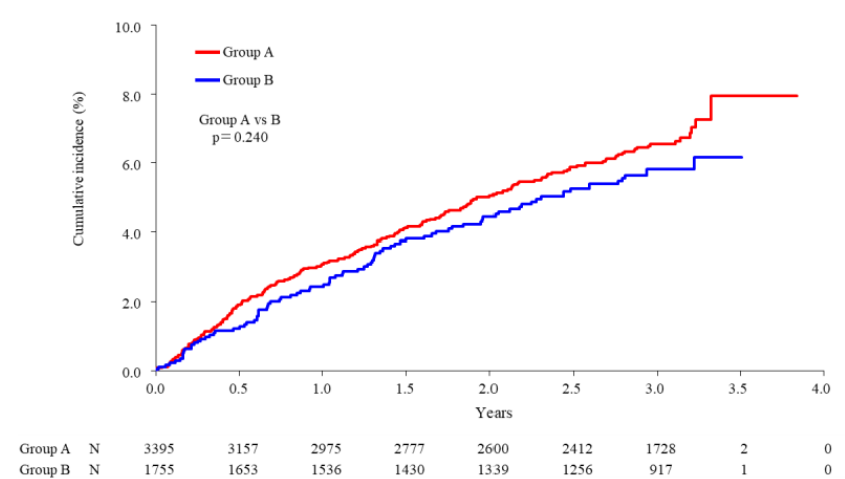

D Cancer

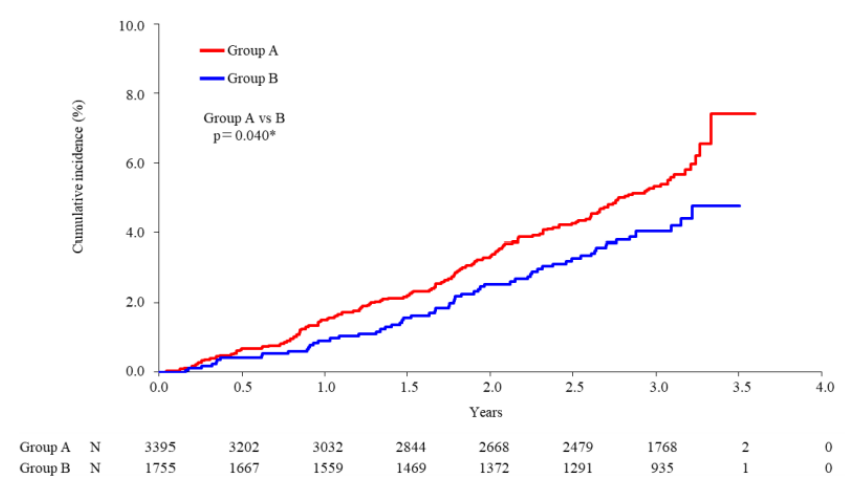

Figure 2 Kaplan-Meier analysis of major adverse events. (A) Symptomatic hypoglycemia, (B) skin disorders of non-extrinsic origin, (C) severe infections, and (D) cancer. Between-group comparisons were performed by log-rank test. Numbers (N) under each time point denote patients at risk.

cumulative incidence of major AEs (see Research design and methods section) is summarized in table 3 with the aid of Kaplan-Meier analysis (figure 2). The difference of the basis for percent incidence calculation should be noted as the Kaplan-Meier method was employed for major AEs (table 3) and microvascular complications (table 4), while the number of patients with AE occurrence was simply divided by SAS population (ie, $\mathrm{n}=3395$ for group A and $n=1755$ for group B) for individual AEs (see online supplemental table 2), macrovascular complications (table 5), and serious adverse events (SAEs; see online supplemental table 2).

\begin{tabular}{|c|c|c|c|c|}
\hline & & Group A (N=3395) & Group B (N=1755) & $P$ value \\
\hline & & n (\%) & n (\%) & Group A vs B \\
\hline \multirow[t]{3}{*}{ Diabetic retinopathy } & Onset/progression & $62(2.3)$ & $27(2.0)$ & 0.455 \\
\hline & Onset & $43(2.0)$ & $21(1.9)$ & 0.687 \\
\hline & Progression & $19(3.6)$ & $6(3.1)$ & 0.661 \\
\hline \multirow[t]{3}{*}{ Diabetic nephropathy } & Onset/progression & $76(3.0)$ & $51(6.0)$ & 0.147 \\
\hline & Onset & $49(2.6)$ & $37(6.2)$ & 0.117 \\
\hline & Progression & $27(4.2)$ & $14(4.9)$ & 0.683 \\
\hline \multirow[t]{3}{*}{ Diabetic neuropathy } & Onset/progression & $30(1.1)$ & $13(1.4)$ & 0.588 \\
\hline & Onset & $21(1.0)$ & $12(1.7)$ & 0.825 \\
\hline & Progression & $9(1.8)$ & $1(0.4)$ & 0.142 \\
\hline
\end{tabular}

Cumulative incidence (\%) of microvascular complications was calculated per observation period by the Kaplan-Meier method (see online supplemental figure 2). P values were by log-rank test. 
Table 5 Summary of symptomatic macrovascular events

\begin{tabular}{|c|c|c|c|c|c|c|}
\hline \multirow{2}{*}{ Category } & & \multicolumn{2}{|c|}{ Group A (N=3395) } & \multicolumn{2}{|c|}{ Group B (N=1755) } & \multirow{2}{*}{$\begin{array}{l}\text { P value } \\
\text { Group A vs B }\end{array}$} \\
\hline & & n (\%) & Event & n (\%) & Event & \\
\hline Overall macrovascular events & & $92(2.71)$ & 109 & $52(2.96)$ & 62 & 0.594 \\
\hline Acute coronary syndrome & & $1(0.03)$ & 1 & $0(0.00)$ & 0 & 1.000 \\
\hline Myocardial infarction & & $17(0.50)$ & 17 & $9(0.51)$ & 12 & 1.000 \\
\hline \multirow[t]{2}{*}{ Stroke } & Cerebral infarction & $29(0.85)$ & 32 & $20(1.14)$ & 20 & 0.363 \\
\hline & Cerebral hemorrhage & $11(0.32)$ & 11 & $3(0.17)$ & 3 & 0.406 \\
\hline Heart failure & & $27(0.80)$ & 33 & $11(0.63)$ & 13 & 0.607 \\
\hline \multirow[t]{2}{*}{ Coronary angioplasty } & $\mathrm{PCl}$ & $2(0.06)$ & 2 & $2(0.11)$ & 3 & 0.609 \\
\hline & CABG & $1(0.03)$ & 1 & $0(0.00)$ & 0 & 1.000 \\
\hline PAD & & $8(0.24)$ & 9 & $7(0.40)$ & 8 & 0.413 \\
\hline Unstable angina & & $3(0.09)$ & 3 & $3(0.17)$ & 3 & 0.416 \\
\hline
\end{tabular}

Macrovascular events were selected on symptomatic basis, but events deemed on test/examination basis were not included. The events selected and categorized in the table were acute coronary syndrome, myocardial infarction (acute myocardial infarction and myocardial infarction), cerebral infarction (brain stem infarction, cerebellar infarction, cerebral infarction, cerebral thrombosis, lacunar infarction, thalamic infarction, and thrombotic cerebral infarction), cerebral hemorrhage (brain stem hemorrhage, cerebellar hemorrhage, cerebral hemorrhage, subarachnoid hemorrhage, thalamus hemorrhage, and putamen hemorrhage), heart failure (cardiac failure, cardiac failure acute, cardiac failure chronic, and cardiac failure congestive), PCl (coronary angioplasty and stent placement), CABG (coronary artery bypass), PAD (peripheral arterial occlusive disease), and unstable angina. Incidence (\%) was calculated as a division of $n$ for individual $A E$ category by either 3395 (group A) or 1755 (group B). P values were based on Fisher's exact test.

$\mathrm{AE}$, adverse event; $\mathrm{CABG}$, coronary artery bypass grafting; $\mathrm{PAD}$, peripheral artery disease; $\mathrm{PCl}$, percutaneous coronary intervention.

Of major AEs, symptomatic hypoglycemia was cumulatively reported in 104 (point estimate: $3.9 \%$ ) group A patients and $45(3.2 \%)$ group $B$ patients with no significant difference $(\mathrm{p}=0.317$; table 3 and figure $2 \mathrm{~A})$. Pancreatitis was reported at a low rate in both groups, as acute type in $5(0.2 \%)$ and $3(0.9 \%)$ patients and chronic type in $2(0.1 \%)$ and 0 patients with no statistical betweengroup difference $(\mathrm{p}=0.861$ and $\mathrm{p}=0.310$, respectively, table 3; Kaplan-Meier plots not shown).

Kaplan-Meier analysis gave a similar profile for skin disorders of non-extrinsic origin observed in 201 (7.9\%) group A patients and $90(6.2 \%)$ group B patients $(\mathrm{p}=0.240$; table 3 and figure 2B). Of the observed skin disorder AEs, skin papilloma was with significant between-group difference $(0.0 \%$ vs $0.2 \%, \mathrm{p}=0.040$; see online supplemental table 2). Bullous pemphigoid has been recently suggested in association of the use of DPP-4 inhibitors. ${ }^{2324}$ This $\mathrm{AE}$ was observed in three group A patients but no group B patients with no significant difference ( $\mathrm{p}=0.556$; see online supplemental table 2).

Severe infections were observed in $71(2.7 \%)$ and 28 $(2.0 \%)$ patients, respectively, and their Kaplan-Meier analysis was with no between-group difference $(\mathrm{p}=0.222$; table 3 and figure $2 \mathrm{C}$ ). Of the observed infections, vulvovaginal candidiasis was with between-group difference $(0.0 \%$ vs $0.2 \%, p=0.040$; see online supplemental table 2).

Cancer occurred more frequently in group A (162 patients; $7.4 \%$ ) than in group B (62 patients; $4.8 \%$ ) with a statistical difference $(\mathrm{p}=0.040$; table 3 and figure 2D). Thyroid, lung, stomach, liver, large intestine, and prostate were the frequent sites for the event (see online supplemental table 2). The incidence of pancreatic cancer was low in both groups $(0.1 \%$ each, $\mathrm{p}=1.000$; see online supplemental table 2). No significant difference was observed between the two groups in the incidence of individual cancer. Multivariate Cox regression analysis showed no significant impact by group, but confirmed an increase of the event as HR $(95 \% \mathrm{CI})=3.34$ (2.32 to 4.81 ; $\mathrm{p}<0.001$ ) for age $\geq 65$ and $<75$ years and 5.54 ( 3.78 to 8.14 ; $p<0.001$ ) for age $\geq 75$ years compared with age $<65$ years. Previous smoking habit was another factor contributing to the event as HR $(95 \% \mathrm{CI})=1.70(1.28$ to $2.27 ; \mathrm{p}<0.001)$ compared with the patients with no smoking history (see online supplemental table 3).

SAEs observed during the study period are summarized in online supplemental table 2. Overall incidence of SAEs was $14.6 \%$ in group A and $12.5 \%$ in group B with a small but significant difference $(\mathrm{p}=0.046)$. The table includes serious ones of the reported major AEs (but without defining limitations such as "symptomatic" for hypoglycemia). These major AE categories were of no significant between-group difference in their incidence except for cancer $(\mathrm{p}=0.037)$. "Other" SAEs were observed in $10.3 \%$ and $9.6 \%$ of patients $(\mathrm{p}=0.463)$. Of those, System Organ Classes (SOCs) of Cardiac disorders, Gastrointestinal disorders, Injury, poisoning, and procedural complications, Metabolism and nutrition disorders, and Nervous system disorders were the categories frequently reported. Serious cholangitis under SOC of Hepatobiliary disorders was reported in one $(0.03 \%)$ group A patient and four $(0.2 \%)$ group B patients with between-group significance $(\mathrm{p}=0.049$, not shown in online supplemental table 2). 
We collected information of microvascular complications (diabetic retinopathy, nephropathy, and neuropathy). No significant between-group difference was detected when the onset and progression were analyzed by the Kaplan-Meier method either in combination or separately (table 4 and online supplemental figure 2). Serious microvascular AEs were observed in $0.1 \%$ and $0.2 \%$ of patients, respectively, with no between-group difference $(\mathrm{p}=0.239$, not shown in online supplemental table 2).

Macrovascular events were tabulated in table 5 on symptomatic basis, but not on test/examination basis. Ninety-two $(2.71 \%)$ group A patients and $52(2.96 \%)$ group B patients developed macrovascular complications with no significant between-group differences in the categorized events.

There were AEs under "Others" category reported with statistical between-group difference (see online supplemental table 2). Their incidence was higher in group B than in group A, except for iron-deficiency anemia. The incidence of AEs under SOC "Renal and urinary disorders" was high in group A compared with group B, but none of the individual AEs under this organ class were with significant between-group difference.

\section{Efficacy of alogliptin}

Since J-BRAND Registry was conducted in the real-world setting, there were patients who received insulin products and/or GLP-1 or related formulations for better glycemic control. Furthermore, the use of DPP-4 inhibitors increased in group B patients (see the section of Disposition and baseline characteristics of patients and online supplemental figure 1). For better clarification of the effectiveness of alogliptin (or other DPP-4 inhibitors), we analyzed the efficacy endpoints mainly in the patient population after excluding those who received insulin products and/or GLP-1 or related formulations (group A) and who received insulin products, GLP-1 or related formulations, and/or DPP-4 inhibitors (group B).

Mean $\mathrm{HbAlc}$ was at $7.58 \%(59 \mathrm{mmol} / \mathrm{mol})$ in group A patients and $7.86 \%(62 \mathrm{mmol} / \mathrm{mol})$ in group B patients at baseline with significant difference $(p<0.001$ by twosample t-test; table 1). The parameter decreased significantly in both groups at 0.5 -year visit $(7.00 \%=53 \mathrm{mmol} /$ $\mathrm{mol}$ and $6.96 \%=53 \mathrm{mmol} / \mathrm{mol}$; $\mathrm{p}<0.001 \mathrm{each}$ by onesample t-test) and then nearly plateaued up to the end of 3-year treatment period (see online supplemental figure $3)$. The decrease was larger in group B patients than in group A patients, for example, $-0.76 \%$ versus $-0.60 \%$ at 0.5 -year visit $(\mathrm{p}<0.001$ by two-sample t-test; figure $3 \mathrm{~A})$.

Blood glucose was determined in each patient under a fasting condition. The mean values were $153.9 \mathrm{mg} / \mathrm{dL}$ in group A patients and $157.2 \mathrm{mg} / \mathrm{dL}$ in group B patients at baseline and significantly decreased at the following visits in either group with changes of -9.9 to $-14.5 \mathrm{mg}$ / $\mathrm{dL}$ and -16.3 to $-18.8 \mathrm{mg} / \mathrm{dL}(\mathrm{p}<0.001$; figure $3 \mathrm{~B})$. No statistical difference was observed between the groups. Mean fasting serum insulin was $9.33 \mu \mathrm{U} / \mathrm{mL}$ in group A patients and $10.46 \mu \mathrm{U} / \mathrm{mL}$ in group B patients at baseline, and showed no considerable changes at the later visits (figure 3C). In addition, homeostasis model assessment (HOMA)-R and HOMA- $\beta$ were exploratorily calculated to assess insulin resistance and insulin secretability in the patients. Mean baseline HOMA-R was 3.58 in group $\mathrm{A}$ and 4.07 in group $\mathrm{B}$ with no significant difference. While group A patients showed a slight change ( -0.52 to $0.01)$ and group B patients showed a significant decrease $(-0.80$ to -1.06$)$ at $1.0,2.0$, and 3.0 years post-baseline (by one-sample t-test), the changes were with no significant difference between the groups throughout the study period. Similarly, mean HOMA- $\beta$ was $47.12 \%$ and $48.61 \%$ with no significant difference at baseline, and its changes were small ( $1.76 \%$ to $15.28 \%$ and $-2.08 \%$ to $5.99 \%)$ with no significant between-group difference.

Urinary albumin and serum creatinine were determined and their ratio (ACR) was calculated. The baseline values were $91.4 \mathrm{mg} / \mathrm{g} \cdot$ Cre and $103.1 \mathrm{mg} / \mathrm{g} \cdot$ Cre, respectively, with no statistical difference $(p=0.229$; table 1$)$. The parameter showed no notable changes throughout the study period in both groups (figure 3D).

\section{Over-time changes in other related parameters}

Changes of body weight, ECG abnormalities, total/highdensity lipoprotein (HDL)/LDL cholesterol, and fasting triglycerides are summarized in online supplemental table 4.

Mean weight was higher in group B patients than in group A patients at baseline $(\mathrm{p}<0.001$, table 1$)$ and then significantly decreased at 0.5 -year and later visits (group A: -0.17 to $-0.81 \mathrm{~kg}$, group B: -0.37 to $-0.92 \mathrm{~kg}$ ). Higher weight of group B patients was throughout the study period. The changes from baseline were not significantly different between the two groups (see online supplemental table 4).

ECG abnormalities were found in $7.3 \%$ of group A patients and $4.4 \%$ of group B patients at baseline with significant difference $\left(\mathrm{p}=0.032\right.$ by $\chi^{2}$ test). However, this between-group difference disappeared with stable percentage of abnormalities at 0.5-year and later visits (see online supplemental table 4).

Mean total cholesterol was higher in group B patients than in group A patients at baseline $(\mathrm{p}<0.001$, table 1$)$, and then significantly decreased at later visits (group A: -5.1 to $-6.4 \mathrm{mg} / \mathrm{dL}$, group B: -6.7 to $-10.8 \mathrm{mg} / \mathrm{dL}$ ). The higher level of total cholesterol was throughout the study period in group B, but the difference of changes from baseline were generally insignificant between the two groups (see online supplemental table 4). HDL cholesterol was not largely different between the two groups with only minor changes throughout the study period (see online supplemental table 4). Mean LDL cholesterol was higher in group B patients than in group A patients at baseline $(p<0.001$, table 1$)$ and then significantly decreased at later visits (group A: -4.9 to $-7.1 \mathrm{mg}$ / $\mathrm{dL}$, group B: -7.3 to $-12.3 \mathrm{mg} / \mathrm{dL}$ ). The higher level of LDL cholesterol in group B patients was up to 2.0-year 
A HbAlc

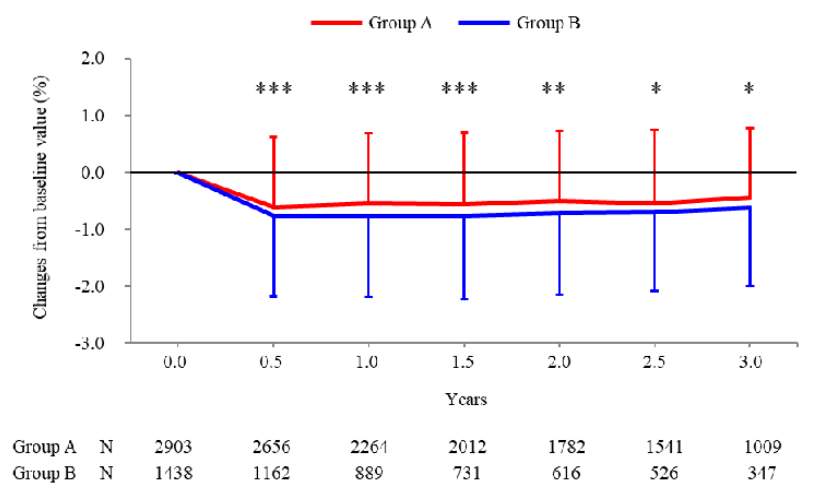

C Fasting insulin

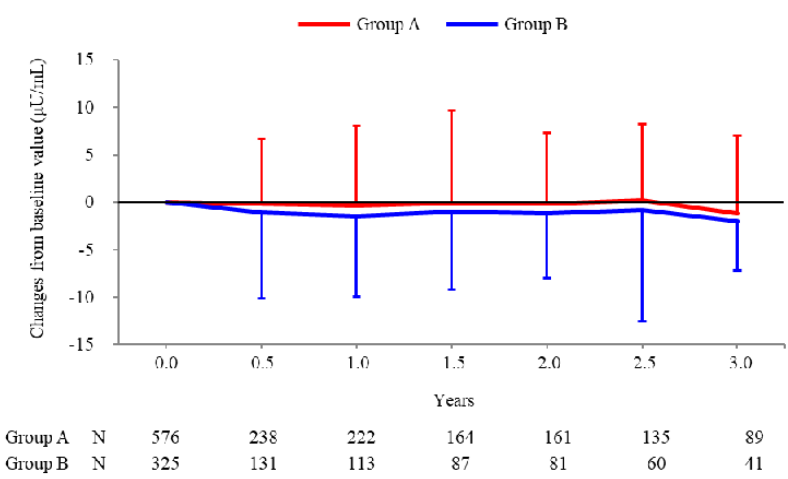

B Fasting glucose

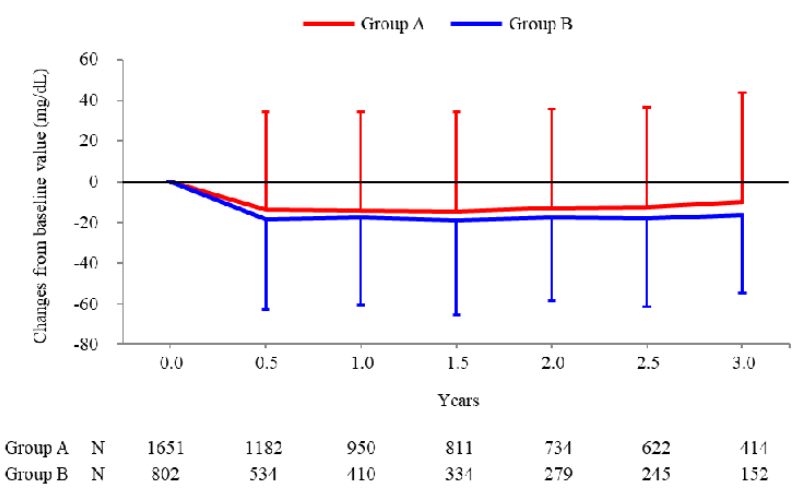

D Urinary albumin

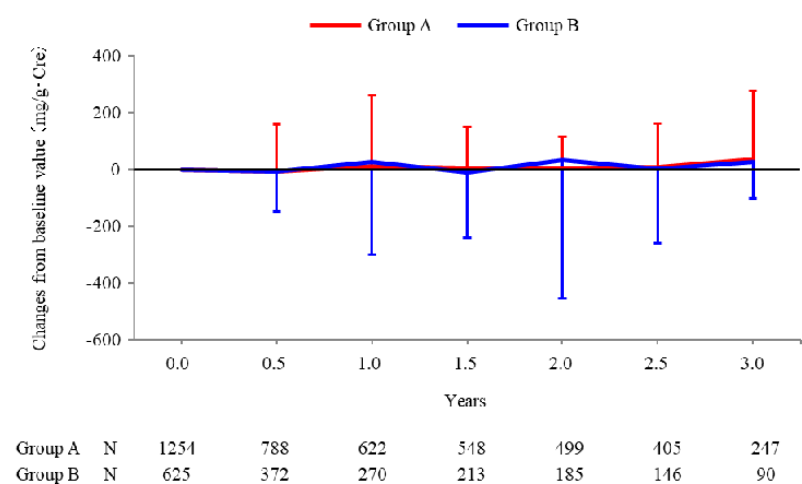

Figure 3 Over-time changes of efficacy endpoints. HbA1c (A), fasting glucose (B), fasting insulin (C), and urinary albumin (D) were determined at baseline and following visits. Changes of these parameters from baseline (mean \pm SD) were plotted against each visit. Note the patients were excluded from the analysis if they received insulin products and/or GLP-1 or related formulations (group A), and received insulin products, GLP-1 or related formulations, and/or DPP-4 inhibitors (group B). Between-group difference was examined at each visit by two-sample t-test, giving significant $\mathrm{p}$ values only for $\mathrm{HbA1c}$ as ${ }^{\star \star *} \mathrm{p}<0.001,{ }^{\star \star \star} \mathrm{p}<0.001,{ }^{\star \star *} \mathrm{p}<0.001,{ }^{\star \star} \mathrm{p}=0.001,{ }^{\star} \mathrm{p}=0.026$, and ${ }^{*} \mathrm{p}=0.027$, respectively. Also note that mean $\mathrm{HbA} 1 \mathrm{c}$ was $7.58 \%$ in group A patients and $7.86 \%$ in group B patients at baseline with significant difference $(p<0.001$ by 2 -sample t-test; table 1$)$. DPP, dipeptidyl peptidase; GLP-1, glucagon-like peptide-1; HbA1c, hemoglobin A1c.

visit and with greater changes from baseline throughout the study period (see online supplemental table 4).

Mean fasting triglycerides was comparable at baseline (table 1). Although the values did not change considerably in group A patients, a significant decrease was observed at 0.5-year, 1.5-year, and 2.0-year visits in group B patients. The difference of this parameter was with no significance between the two groups throughout the study period (see online supplemental table 4 ).

\section{DISCUSSION}

J-BRAND Registry was conducted in patients with type 2 diabetes as a large-scale, multicenter, controlled, prospective, observational study. Given that DPP-4 inhibitors have been extensively used in the patients with the disease during the recent decade, the study constructed a realworld database on the safety and efficacy of the drug class particularly focusing on alogliptin as a representative. ${ }^{16}$
Group A patients $(\mathrm{n}=3395)$ started the study treatment with alogliptin and group B patients $(n=1755)$ with non-DPP-4 inhibitor OHAs. Of patient backgrounds, age, disease duration, BMI, and so on were different between the two groups. These differences reflected the reasonable drug choice based on the pathogenesis and background of the individual patients by the diabetes specialists participating in this study; DPP-4 inhibitors are suitable for non-obese, older patients whose major pathogenesis is likely to be a defect in insulin secretion (especially in the population of East Asian region including Japan), while biguanides are effective for obese, nonelderly patients who are likely to exhibit insulin resistance. Of baseline parameters, blood pressure, HbA1c, weight, total cholesterol, LDL cholesterol, and casual blood glucose were lower in group A patients than in group B patients. 
Of the major safety events, symptomatic hypoglycemia, pancreatitis, skin disorders of non-extrinsic origin, and severe infections were not statistically different in their incidence between the two groups. A risk for pancreatitis, which has been suggested for DPP-4 inhibitors, ${ }^{192}$ was not detected as previously reported regarding alogliptin and other DPP-4 inhibitors by relatively short-term observations. ${ }^{57-10}$ Bullous pemphigoid is another concern, ${ }^{20232426}$ given that DPP-4 inhibitor-related bullous pemphigoid has been reported to preferentially occur in elderly patients treated for several years. Although our study did not detect the increased risk with DPP-4 inhibitor treatment, further study might be needed to draw a definitive conclusion. While skin papilloma and vulvovaginal candidiasis were exceptionally observed in group B with a statistical significance over group A, their incidence was low and none of those were reported as treatment related.

Cancer was observed more frequently in group A than in group B, while the incidence of individual cancer was not different. The between-group difference of all cancers was attributable to group A patients being significantly older than group B patients. Presumably due to a similar reason, there was a small increase of SAEs including cancers in group A compared with those in group B. It should be noted the incidence of pancreatic cancer was low in both groups.

Onset and progression of microvascular complications were reported at comparable rates between the two groups when analyzed either in combination or separately. The incidence similarity was also observed for macrovascular events between the groups. Several outcome studies showed no increase in cardiovascular risk by DPP-4 inhibitors. ${ }^{7-10}$ The current study confirmed the safe profile of alogliptin in terms of cardiovascular disease in the real-world setting. It should be noted that alogliptin use achieved the similar glycemic control and showed the similar incidence of macrovascular complications to that observed in the group predominantly using metformin, which has been shown to suppress macrovascular complications. ${ }^{27}$ Previous studies raised some concern about the risk of heart failure by DPP-4 inhibitor use. $^{7812}$ While recent studies have shown the protective effects of SGLT2 inhibitors for heart failure, ${ }^{28}$ SGLT2 inhibitor use was very low in both groups and alogliptin use was not associated with the risk in the current study, confirming the safe profile of alogliptin for heart failure.

The low risk of weight gain was also confirmed for DPP-4 inhibitors during long passage of the treatment of patients with diabetes mellitus.

The efficacy profile was similar between the two groups as observed for the time-dependent decrease in HbAlc and fasting blood glucose. Importantly, group A patients achieved and maintained the target HbAlc $(7.0 \%=53 \mathrm{mmol} / \mathrm{mol})$ in average throughout the study with only a slight increase in the number of medications (the mean number of drugs used: from 2.1 to 2.3), while group B patients also maintained the target but with more increase in the number of medications (from 1.6 to 2.0). Moreover, alogliptin use appeared to preserve the ability of insulin secretion as evidenced by the maintained HOMA- $\beta$ for 3-year study period, different from the decline by sulfonylurea-based therapy in the United Kingdom Prospective Diabetes Study, ${ }^{29}$ while group B patients using metformin as a major medication showed no decrease in HOMA- $\beta$, either, as shown by the previous study. ${ }^{30}$ The effect of alogliptin on albuminuria was not different from that observed in group B, suggesting that achieving the target HbA1c is important for the suppression of diabetic kidney disease rather than using specific medication.

DPP-4 inhibitors are widely used in Japan and other East Asian countries since the incretin-based therapy is more efficacious for the Asian subjects than Caucasians. ${ }^{31}$ The current study has shown that DPP-4 inhibitors are more preferentially prescribed to relatively older and thinner patients with longer disease history in the real-world setting as expected. Prevalence of the elderly patients with diabetes has been and will be increasing, ${ }^{32}$ and safety profile of medication including low risk of severe hypoglycemia ${ }^{33}$ tightly associated with cognitive decline and frailty is important. With this regard, the current study provides the evidence of the safety of DPP-4 inhibitors. It also suggests that the treatment of elderly patients with DPP-4 inhibitors is durable for glucose-lowering and maintenance of the pancreatic $\beta$-cell function.

On the other hand, the current study has certain limitations: (1) differences were observed in patients' baseline characteristics, (2) no centralized adjudication system was applied to the safety and efficacy evaluations, and thereby the collection of macrovascular events in particular may have been influenced. These limitations are, however, a reflection of real-world context of type 2 diabetes treatment. Nevertheless, the current study for the first time demonstrated in a prospective fashion that alogliptin is not associated with any risks previously concerned and is durable in terms of glucose-lowering compared with the biguanide-based therapy.

In conclusion, alogliptin, as a representative of DPP-4 inhibitors, was revealed as a safe and efficacious agent for the treatment of patients with type 2 diabetes in the realworld setting.

\section{Author affiliations}

${ }^{1}$ National Center for Global Health and Medicine, Diabetes Research Center, Tokyo, Japan

${ }^{2}$ Yamaguchi University Graduate School of Medicine, Ube, Yamaguchi, Japan

${ }^{3}$ Aichi Medical University School of Medicine, Nagakute, Aichi, Japan

${ }^{4}$ Akita University Graduate School of Medicine, Akita, Japan

${ }^{5}$ Kyoto University Graduate School of Medicine, Kyoto, Japan

${ }^{6}$ Juntendo University Graduate School of Medicine, Tokyo, Japan

${ }^{7}$ Osaka University Graduate School of Medicine, Osaka, Japan

${ }^{8}$ Jikei University School of Medicine, Tokyo, Japan

${ }^{9}$ Hokkaido University Graduate School of Medicine, Sapporo, Japan

${ }^{10}$ Asahikawa Medical University, Asahikawa, Hokkaido, Japan

${ }^{11}$ Tohoku University Graduate School of Medicine, Sendai, Japan

${ }^{12}$ Kanto Medical Center NTT EC, Tokyo, Japan

${ }^{13}$ Saitama Medical University Faculty of Medicine, Moroyama, Saitama, Japan 
${ }^{14}$ Aichi Gakuin University School of Dentistry, Nagoya, Japan

${ }^{15}$ Kochi University Medical School, Nankoku, Kochi, Japan

${ }^{16}$ Saijo Central Hospital, Saijo, Ehime, Japan

${ }^{17}$ Center for Innovative Clinical Medicine, Okayama University Hospital, Okayama, Japan

${ }^{18}$ University of Occupational and Environmental Health School of Medicine,

Kitakyushu, Fukuoka, Japan

${ }^{19}$ Kumamoto University, Faculty of Life Sciences, Kumamoto, Japan

${ }^{20} \mathrm{Graduate}$ School of Medicine, International University of Health and Welfare,

Tokyo, Japan

${ }^{21}$ Department of Diabetes and Metabolic Diseases, Graduate School of Medicine, The University of Tokyo, Tokyo, Japan

${ }^{22}$ Toranomon Hospital, Federation of National Public Service Personnel Mutual Aid Associations, Tokyo, Japan

Acknowledgements The authors sincerely acknowledge Dr. M. Kasuga (Head of the Institute for Adult Diseases, Asahi Life Foundation, Tokyo) and Professor H. Sakura (Head of Internal Medicine, Tokyo Women's Medical University Medical Center East, Tokyo) for independently assessing the safety observations throughout the study. We thank A2 Healthcare Corporation (Tokyo) for administrative, clinical monitoring, and data monitoring activities; AC Medical (Tokyo) for statistical analyses; and CMIC Co., Ltd. (Tokyo) and its employee, Dr. Y. Takeuchi for preparation and submission of the manuscript. We express deepest acknowledgments to Takeda Pharmaceutical Co., Ltd. for funding all expenses required for the conduct of J-BRAND Registry. It should be noted the company and its employees were never involved in any procedures of this project.

Collaborators Hideaki Miyoshi, Hokkaido University Hospital; Atsuko Abiko, Asahikawa Medical University Hospital; Sou Nagai, NTT-East Sapporo Hospital; Katsuyuki Yanagisawa, Sapporo City General Hospital; Hiromichi Kijima, Tonan Hospital; Shinji Taneda, Manda Memorial Hospital; Shigeyuki Saitoh, Sapporo Medical University Hospital; Daisuke Ikeda, Chitose City Hospital; Fuminori Hirano, Asahikawa Medical Center; Takanori Miura, Asahikawa-Kosei General Hospital; Yoshihiro Miyamoto, Asahikawa City Hospital; Haruhiko Yoshimura, Iwamizawa Municipal General Hospital; Mitsutaka Inoue, Engaru-Kosei General Hospital; Masahiko Katoh, Hokkaido Medical Center; Osamu Nakagaki, Tomakomai City Hospital; Chiho Yamamoto, Tomakomai City Hospital; Akitsuki Morikawa, Japanese Red Cross Asahikawa Hospital; Kazuhiro Yoshida, Tenshi Hospital; Shin Furukawa, Kushiro Red Cross Hospital; Takeshi Koshiya, Obihiro-Kosei Hospital; Hajime Sugawara, Obihiro-Kosei Hospital; Takumi Uchida, Obihiro-Kosei Hospital; Yuichiro Yamada, Akita University Hospital; Hideki Katagiri, Tohoku University Hospital; Hiroshi Yamaguchi, Yamagata Saisei Hospital; Noe Takakubo, Yamagata Saisei Hospital; Yasushi Ishigaki, Iwate Medical University Hospital; Susumu Suzuki, Ohta General Hospital Foundation Ohta Nishinouchi Hospital; Takashi Shimotomai, Akita Kousei Medical Center; Naoki Tamasawa, Aomori City Hospital; Jun Matsui, Aomori City Hospital; Takashi Goto, Japanese Red Cross Akita Hospital; Toshihide Oizumi, Yamagata University Hospital; Shinji Susa, Yamagata University Hospital; Makoto Daimon, Yamagata University Hospital; Jun Matsui, Hirosaki University Hospital; Hiroshi Murakami, Hirosaki University Hospital; Takashi Sugawara, Iwate Prefectural Central Hospital; Hiroaki Akai, Tohoku Rosai Hospital; Masahiro Matsumoto, Tohoku Rosai Hospital; Mari Nakamura, Tohoku Rosai Hospital; Yoshiji Ogawa, Aomori Prefectural Central Hospital; Takao Yokoshima, Iwate Prefectural Karumai Hospital; Tsuyoshi Watanabe, Fukushima Medical University Hospital; Hiroaki Satoh, Fukushima Medical University Hospital; Michio Shimabukuro, Fukushima Medical University Hospital; Kazuhisa Tsukamoto, Fukushima Medical University Aizu Medical Center; Motoei Kunimi, Fukushima Medical University Aizu Medical Center; Jo Satoh, Tohoku Medical and Pharmaceutical University Wakabayashi Hospital; Atushi Okuyama, Yokote Municipal Hospital; Kazutaka Ogawa, Yokote Municipal Hospital; Hideyuki Eguchi, Okitama Public General Hospital; Mamoru Kimura, Nihonkai General Hospital; Hiroshi Kouno, Jusendo General Hospital; Yohei Horikawa, Hiraka General Hospital; Shin Ikejima, Odate Municipal General Hospital; Masaru Saitoh, Kureha General Hospital; Naoyoshi Minami, Osaki Citizen Hospital; Akihiro Sekikawa, Shiogama City Hospital; Ryo Suzuki, The University of Tokyo Hospital; Toyoyoshi Uchida, Juntendo University Hospital; Rimei Nishimura, The Jikei University Hospital; Michio Hayashi, NTT Medical Center Tokyo; Akira Shimada, Tokyo Saiseikai Central Hospital; Toshihide Kawai, Tokyo Saiseikai Central Hospital; Nobuya Fujita, Saiseikai Utsunomiya Hospital; Ken Tomotsune, Saiseikai Utsunomiya Hospital; Shigeo Yamashita, JR Tokyo General Hospital; Motoji Naka, Asama General Hospital; Toru Hiyoshi, Japanese Red Cross Medical Center; Yoshiyuki Nagai, Tokyo Medical University Hospital; Tomotaka Katoh, Tokyo Medical University Hospital; Kumiko Hamano, Kanto Rosai Hospital; Kouichi Inukai, Kyorin University Hospital; Takuma Kondo,
Kyorin University Hospital; Kazuhiro Tsumura, Kawasaki Municipal Hospital; Yoko Matsuzawa, Yokohama Rosai Hospital; Masahiro Mimura, Chiba Rosai Hospital; Masahiko Kawasumi, Juntendo University Nerima Hospital; Izumi Takei, Tokyo Dental College Ichikawa General Hospital; Yoshiaki Okubo, Tokyo Dental College Ichikawa General Hospital; Masafumi Matsuda, Saitama Medical University Saitama Medical Center; Ichiro Tatsuno, Toho University Medical Center Sakura Hospital; Nobuyuki Banba, Dokkyo Medical University Nikko Medical Center; Satoru Yamada, Kitasato University Kitasato Institute Hospital; Akihiko Ando, Jichi Medical University Hospital; Masao Toyoda, Tokai University Hospital; Daisuke Suzuki, Tokai University Hospital; Takahiro lijima, Yokohama City University Hospital; Yasumichi Mori, Toranomon Hospital; Yutaka Uehara, Japanese Red Cross Maebashi Hospital; Yoshihiko Satoh, Shinshu University Hospital; Hitoshi Shimano, University of Tsukuba Hospital; Akihiro Isogawa, Mitsui Memorial Hospital; Kazuaki Yahata, Nagaoka Chuo General Hospital; Yoshimasa Asoh, Dokkyo Medical University Hospital; Koichiro Kuwabara, Tokyo Rosai Hospital; Souichi Takizawa, University of Yamanashi Hospital; Yasushi Tanaka, St. Marianna University School of Medicine Hospital; Koutaroh Yokote, Chiba University Hospital; Masako Tohgo, Tokyo Takanawa Hospital; Takanobu Itoi, Tokyo Takanawa Hospital; Shigeru Miyazaki, Shin-Yamanote Hospital; Hiroshi Itoh, Keio University Hospital; Teruo Shiba, Toho University Ohashi Medical Center; Takahisa Hirose, Toho University Omori Medical Center; Mariko Higa, Saiseikai Yokohamashi Tobu Hospital; Masanobu Yamada, Gunma University Hospital; Osamu Ogawa, Kameda Medical Center; Masatoshi Kuroki, Nerima Hikarigaoka Hospital; Shinobu Satoh, Chigasaki Municipal Hospital; Makoto Ujihara, Yokohama Medical Center; Kenjiroh Yamanaka, Sasaki Foundation Kyoundo Hospital; Hajime Koyano, Juntendo University Urayasu Hospital; Tadashi Yamakawa, Yokohama City University Medical Center; Yoshihito Atsumi, Life Extension Laboratory Eiju General Hospital; Kenichiroh Takahashi, Yokohama Minami Kyousai Hospital; Kazuki Orime, Yokohama Minami Kyousai Hospital; Tsutomu Hirano, Showa University Hospital; Jiroh Morimoto, JCHO Saitama Medical Center; Takuya Awata, Saitama Medical University Hospital; Takashi Itoh, Kashima Rosai Hospital; (closed, and reorganised with Kamisu Saiseikai Hospital, May 2019); Yuzoh Mizuno, Kanto Central Hospital; Naoyuki Yamamoto, Tokai University Hachioji Hospital; Han Miyatake, Tokai University Hachioji Hospital; Mina Yamaguchi, Hiratsuka Kyosai Hospital; Hirohito Sone, Niigata University Medical and Dental Hospital; Kenji Yamane, Tamakyuryo Hospital; Masahiko Kure, AOI Universal Hospital; Satoko Kawabe, Ushiku Aiwa General Hospital; Masahumi Kakei, Jichi Medical University Saitama Medical Center; Masashi Yoshida, Jichi Medical University Saitama Medical Center; Hiroyuki Itoh, Edogawa Hospital; Nobuaki Minami, Kinugasa Hospital; Kazuki Kobayashi, Kokuho Asahi General Hospital; Yusuke Fujino, New Tokyo Hospital; Makoto Shibuya, Yokosuka Kyosai Hospital; Kazuki Fukui, Kanagawa Cardiovascular and Respiratory Center; Midori Hosokawa, JCHO Yokohama Chuo Hospital; Isao Nozaki, Koto Hospital; Chigure Nawa, Tokyo Kyosai Hospital; Tamio leiri, International University of Health and Welfare Shioya Hospital; Takayuki Watanabe, Yokohama City Minato Red Cross Hospital; Yoshio Katoh, Kanagawa Prefectural Ashigarakami Hospital; Takuyuki Katabami, St.Marianna University School of Medicine, Yokohama City Seibu Hospital; Michiko Handa, Kawasaki Municipal Ida Hospital; Issei Shimada, Shimada General Hospital; Kenichi Ohya, Shin-Oyama City Hospital; Yoshihiro Ogawa, Tokyo Medical and Dental University Medical Hospital; Takanobu Yoshimoto, Tokyo Medical and Dental University Medical Hospital; Jiroh Nakamura, Aichi Medical University Hospital; Keiko Naruse, Aichi Gakuin University Dental Hospital; Fumihiko Sato, Juntendo University Shizuoka Hospital; Junko Sato, Juntendo University Shizuoka Hospital; Naotsuka Okayama, Nagoya City University Hospital; Kenro Imaeda, Nagoya City University Hospital; Syuko Yoshioka, Tosei General Hospital; Masako Murakami, Japanese Red Cross Shizuoka Hospital; Takashi Murase, Nagoya Medical Center; Yoshihiko Yamada, International University of Health and Welfare Atami Hospital; Yutaka Yano, Mie University Hospital; Hiromitsu Sasaki, Kasugai Municipal Hospital; Yasuhiro Sumida, JCHO Yokkaichi Hazu Medical Center; Kohei Ogawa, Asahi Rousai Hospital; Osamu Yonaha, Yonaha General Hospital; Hiroshi Sobajima, Ogaki Municipal Hospital; Mitsuyasu Ito, Fujita Health University Hospital; Atushi Suzuki, Fujita Health University Hospital; Atsuko Ishikawa, Nishichita General Hospital; Takehiko Ichikawa, Kuwana City Medical Center; Shogo Asano, Toyokawa City Hospital; Makoto Nakamura, Toyokawa City Hospital; Shinobu Goto, Nagoya Memorial Hospital; Sakuma Hiroya, Nagoya Memorial Hospital; Hiroshi Murase, Daiyukai General Hospital; Shozo Ogawa, Tokai Central Hospital; Hideki Okamoto, Meitetsu Hospital; Kotaro Nagai, Hashima City Hospital; Koji Nagayama, Hamamatsu Medical Center; Masanori Yoshida, Nagoya Ekisaikai Hospital; Norio Takahashi, Nagoya Ekisaikai Hospital; Kazuhisa Takami, Kizawa Memorial Hospital; Tsuneo Ono, Inazawa Kosei Hospital; Takanobu Morihiro, Masuko Memorial Hospital; Daisuke Tanaka, Kyoto University Hospital; lichiro Shimomura, Osaka University Hospital; Noriko Takahara, Ako City Hospital; Satoshi Miyata, JCHO Osaka Hospital; Mamiko Tsugawa, Ikeda City Hospital; Koichiro 
Yasuda, Saiseikai Noe Hospital; Seiji Muro, Osaka Red Cross Hospital; Masanori Emoto, Osaka City University Hospital; Daisuke Koya, Kanazawa Medical University Hospital; Ikuo Mineo, Toyonaka Municipal Hospital; Ichiro Shiojima, Kansai Medical University Hospital; Takeshi Kurose, Kansai Electric Power Hospital; Takashi Akamizu, Wakayama Medical University Hospital; Makoto Ohashi, Osaka Rosai Hospital; Yumiko Kawabata, Kindai University Hospital; Mitsushige Nishikawa, Kansai Medical University Medical Center; Emiko Nomura, Kansai Medical University Medical Center; Yasuyuki Nishimura, Japanese Red Cross Kanazawa Hospital; Yasuhiro Ono, Kindai University Sakai Hospital; Yasuhisa Yamamoto, Wakayama Rosai Hospital; Keigo Naka, Wakayama Rosai Hospital; Kazuhiko Sakaguchi, Kobe University Hospital; Taizo Yamamoto, Kyoto Katsura Hospital; Rika Usuda, Toyama Prefectural Central Hospital; Hiroshi Akahori, Toyama Prefectural Central Hospital; Seika Kato, Hirakata Kohsai Hospital; Akihito Otsuka, Kawasaki Hospital; Hiroshi Maegawa, Shiga University of Medical Science Hospital; Masahiro Yamazaki, University Hospital Kyoto Prefectural University of Medicine; Hiroyuki Konya, Ashiya Municipal Hospital; Yutaka Umayahara, Osaka General Medical Center; Takashi Seta, Saiseikai Kanazawa Hospital; Hideki Taki, Osaka National Hospital; Masashi Sekiya, Gokeikai Osaka Kaisei Hospital; Naoki Matsuoka, Kobe City Medical Center General Hospital; Shinichi Mogami, Saiseikai Suita Hospital; Sumie Fujii, Ishikawa Prefectural Central Hospital; Toshiyuki Hibuse, Suita Municipal Hospital; Shingo Tsuji, JCHO Osaka Minato Central Hospital; Hirofumi Sumi, Kanazawa University Hospital; Yasuro Kumeda, Minami-Osaka Hospital; Akinori Kogure, Kyoto City Hospital; Kenji Furukawa, JCHO Kanazawa Hospital; Akira Kuroe, Hikone Municipal Hospital; Hideaki Sawaki, Arisawa General Hospital; Narihiro Hibiki, Arisawa General Hospital; Yoshihiro Kitagawa, Osaka General Hospital of West Japan Railway Company; Yukihiro Bando, Fukui-ken Saiseikai Hospital; Akira Ono, Rinku General Medical Center; Rikako Uenaka, Otemae Hospital; Seitaro Omoto, Korigaoka Yukeikai Hospital; Yuki Kita, Tonami General Hospital; Eiko Ri, Izumiotsu Municipal Hospital; Ryutaro Numaguchi, Izumiotsu Municipal Hospital; Sachiko Kawashima, Tango Central Hospital; Ichiro Kisimoto, National Cerebral and Cardiovascular Center Hospital; Kiminori Hosoda, National Cerebral and Cardiovascular Center Hospital; Yoshihiko Araki, Osaka Habikino Medical Center; Tetsuroh Arimura, JCHO Shiga Hospital; Mitsuru Hashiramoto, Yodogawa Christian Hospital; Koumei Takeda, Yamaguchi University Hospital; Akira Matsutani, Shunan City Shinnanyo Hospital; Kenichi Shikata, Okayama University Hospital; Yasushi Inoue, Yamaguchi Prefectural Grand Medical Center; Fumio Sawano, Hiroshima Red Cross Hospital \& Atomic-bomb Survivors Hospital; Nozomu Kamei, Hiroshima Red Cross Hospital \& Atomic-bomb Survivors Hospital; Yasuo Ito, Shimane Prefectural Central Hospital; Miwa Morita, Shimane University Hospital; Yoshiaki Oda, Higashihiroshima Medical Center; Rui Kishimoto, Higashihiroshima Medical Center; Katsuhiro Hatao, JCHO Tokuyama Central Hospital; Mitsuru Hashiramoto, Kawasaki Medical School Hospital; Tomoatsu Mune, Kawasaki Medical School Hospital; Fumiko Kawasaki, Kawasaki Medical School General Medical Center; Hiroki Teragawa, JR Hiroshima Hospital; Ken Yaga, Yamaguchi Rosai Hospital; Keita Ishii, Chugoku Central Hospital; Kyouji Hirata, Chugoku Central Hospital; Tatsuaki Nakatou, Okayama Saiseikai General Hospital; Yutaka Nitta, Shimonoseki Saiseikai General Hospital; Naoki Fujita, Saiseikai Yamaguchi Hospital; Masayasu Yoneda, Hiroshima University Hospital; Masatoshi Tsuru, Kanmon Medical Center; Shinichirou Ando, Okayama City Hospital; Toshiaki Kakiba, Matsue Red Cross Hospital; Michihiro Toyoshige, Sanyo-onoda Municiple Hospital; Tsuguka Shiwa, Hiroshima City Asa Citizens Hospital; Masazumi Fujiwara, Saijo Central Hospital; Hiroaki Miyaoka, Saiseikai Matsuyama Hospital; Hitomi Imachi, Kagawa University Hospital; Yasumi Shintani, Tokushima Red Cross Hospital; Takenori Sakai, Yawatahama City General Hospital; Tetsuji Niiya, Matsuyama Shimin Hospital; Shinpei Fujimoto, Kochi Medical School Hospital; Hisaka Minami, Ehime Prefectural Niihama Hospital; Yoshihiko Noma, Kawashima Hospital; Masaaki Tamaru, Saiseikai Imabari Second Hospital; Yoshitaka Sayou, Takamatsu Red Cross Hospital; Tomoyo Oyama, Takamatsu Red Cross Hospital; Masamoto Torisu, Saiseikai Saijo Hospital; Yuichi Fujinaka, Tokushima Prefecture Naruto Hospital; Yoshitaka Kumon, Chikamori Hospital; Shozo Miyauchi, Uwajima City Hospital; Haruhiko Osawa, Ehime University Hospital; Morikazu Onji, Saiseikai Imabari Hospital; Sumitomo Besshi Hospital, Toru Nakamura; Yoichi Hiasa, Ehime University Hospital; Yousuke Okada, Hospital of the University of Occupational and Environmental Health; Toshihiko Yanase, Fukuoka University Hospital; Kenro Nishida, Minamata City General Hospital \& Medical Center; Syuji Nakamura, Heiwadai Hospital; Takashi Doi, Saga-Ken Medical Centre Koseikan; Kunihisa Kobayashi, Fukuoka University Chikushi Hospital; Nobuhiko Wada, Kurume University Hospital; Moritake Higa, Tomishiro Central Hospital; Koji Matsushita, Wakamatsu Hospital of the University of Occupational and Environmental Health; Yoshihiko Nishio, Kagoshima University Hospital; Ryoji Fujimoto, Shin-Kokura Hospital; Yasuyuki Kihara, Kitakyushu General Hospital; Shinichiro Mine, Sasaki Hospital; Tadashi Arao, Kyushu Rosai Hospital Moji Medical Center; Hiromi Tasaki, Kitakyushu City Yahata Hospital; Yasuto Matsuo, Saiseikai
Kumamoto Hospital; Hirofumi Matsuda, Tamana Central Hospital; Kohei Uriu, Nakama Municipal Hospital; Masakazu Kobayashi, Nagasaki University Hospital; Kazuko Kanda, Saiseikai Yahata General Hospital; Kazuo Ibaraki, Karatsu Red Cross Hospital; Yoshio Kaku, Ashiya Central Hospital; Yasuhiro Takaki, Ashiya Central Hospital; Iwaho Hazekawa, Kumamoto City Hospital; Kenji Ebihara, Kumamoto Saishunso National Hospital; Eiichiro Watanabe, Kumamoto Saishunso National Hospital; Iku Sakurada, Kumamoto Saishunso National Hospital; Kazuhisa Muraishi, Omuta City Hospital; Tamami Oshige, Omuta City Hospital; Junichi Yasuda, Omuta City Hospital; Toyoshi Iguchi, Kyushu University Hospital; Noriyuki Sonoda, Kyushu University Hospital; Masahiro Adachi, Kokura Medical Center; Isao Ichino, Kokura Medical Center; Yuko Horiuchi, Kokura Medical Center; Udai Nakamura, Kyushu University Hospital; Souichi Uekihara, Japanese Red Cross Kumamoto Hospital; Shingo Morimitsu, Imakiire General Hospital; Mitsuhiro Nakazawa, Nanpuh Hospital; Tadashi Seguchi, Oita Prefectural Hospital; Kengo Kaneko, Kumamoto Rosai Hospital; Yasuhiro Ono, Takagi Hospital.

Contributors All authors were the members of Area-assigned Chief Committee and periodically gathered to review the status of study progress. $\mathrm{KU}$ organized the Administrative Bureau to steer the study progress. NI, HW, and YT designed the study protocol. YT was also responsible for data handling. JN, YY, RN, and IS were responsible for the judgment of data for publication. TY was responsible for statistical analysis. TK is the guarantor of entire works associated with the present study and takes responsibility for the integrity of the data and the accuracy of data analysis. TK and $\mathrm{KU}$ extensively contributed to the manuscript preparation. All authors including $\mathrm{HM}, \mathrm{AA}, \mathrm{HK}, \mathrm{MH}, \mathrm{AS}, \mathrm{KN}, \mathrm{SF}, \mathrm{MF}, \mathrm{KS}, \mathrm{YO}$, and EA reviewed and approved the manuscript before submission.

Funding This study was an investigator-initiated study, conducted according to the written agreement signed by Takeda Pharmaceutical Co., Ltd., the principal investigator of this study (TK), and the participating investigators who were directed to perform administrative activities, monitoring, and collection and analysis of data. The corresponding author (TK) had full access to all the study data and had final responsibility for the decision to submit for publication.

Disclaimer The funder was in no involvement with any study procedures including study design and data collection/analysis/interpretation.

Competing interests KU reports lecture fees from Takeda, Novo Nordisk, Nippon Boehringer Ingelheim, Mitsubishi Tanabe Pharma, AstraZeneca, MSD, Ono, Sumitomo Dainippon Pharma, Sanofi, and Astellas; research grants from Astellas, Novo Nordisk, Eli Lilly, Nippon Boehringer Ingelheim, Abbott Japan, and MSD; endowments from Takeda, Astellas, Novo Nordisk, Sumitomo Dainippon Pharma, Sanofi, Kyowa Hakko Kirin, Mitsubishi Tanabe Pharma, Daiichi-Sankyo, and Ono. YT reports lecture fees from Takeda, Astellas, AstraZeneca, MSD, Ono, Kyowa Hakko Kirin, Kissei, Kowa, Sanofi Aventis, from Taisho Pharmaceutical, Taisho Pharma, Mitsubishi Tanabe Pharma, Nippon Shinyaku, Bristol-Myers Squibb, Sumitomo Dainippon Pharma, Chugai, Eli Lilly, Nippon Boehringer Ingelheim, and Novo Nordisk; manuscript fees from Kyowa Hakko Kirin; consulting fees from Terumo; research grants from Daiichi-Sankyo and Novo Nordisk; endowments from Astellas, Takeda, Chugai, Sanwa Kagaku, Daiichi-Sankyo, MSD, Kyowa Hakko Kirin, Eisai, Sumitomo Dainippon Pharma, Shionogi, Eli Lilly, Sanofi Aventis, Mitsubishi Tanabe Pharma, Novo Nordisk, Kissei, Nippon Boehringer Ingelheim, Kowa Pharmaceutical, Teijin Pharma, Pfizer, Nihon Pharmaceutical, and Nippon Shinyaku. JN reports lecture fees from Takeda, Astellas, AstraZeneca, MSD, Ono, Kowa Pharmaceutical, Sanofi, Daiichi-Sankyo, Mitsubishi Tanabe Pharma, Terumo, Eli Lilly, Nippon Boehringer Ingelheim, Novartis, Novo Nordisk, Japan Tobacco Inc., Taisho Pharma, Sumitomo Dainippon Pharma, Sanwa Kagaku, Kissei, Mylan, Arkray, Medtronic Japan, Fukuda Denshi, and Abbott Japan; research grants from Kissei, Nippon Boehringer Ingelheim, and Fukuda Colin; endowments from Takeda Astellas, MSD, Ono, Daiichi-Sankyo, Taisho Pharma, Sumitomo Dainippon Pharma, Mitsubishi Tanabe Pharma, Eli Lilly, Novartis, Novo Nordisk, Japan Tobacco Inc., Otsuka Pharmaceutical, Kaken Pharmaceutical, Kissei, Kyowa Hakko Kirin, Kowa Pharmaceutical, Sanwa Kagaku, Shionogi, Nippon Boehringer Ingelheim, Nippon Becton Dickinson, and Pfizer. YY reports lecture fees from MSD, Novo Nordisk, Ono, Sumitomo Dainippon Pharma, Mitsubishi Tanabe Pharma, Takeda, Sanofi, and Daiichi-Sankyo; endowments from Ono, Takeda, Mitsubishi Tanabe Pharma, Daiichi-Sankyo, and Sanwa Kagaku. NI reports lecture fees from Takeda, Kowa Pharmaceutical, MSD, Arkray, Astellas, Kissei, Sanofi, Novartis, Novo Nordisk, Bayer, Ono, Kyowa Hakko Kirin, Sumitomo Dainippon Pharma, Daiichi-Sankyo, Eli Lilly, Nippon Boehringer Ingelheim, Mitsubishi Tanabe Pharma, Medtronic Japan, Taisho Pharma, Pfizer, Fujifilm Pharma, Saishin Igaku Co., Toyooka Hospital, Terumo, Olympus, Sunstar Foundation, Japan Tabacco Inc., ILSI Japan, University of Occupational and Environmental health, Japan, Japanese Red Cross Wakayama Medical Center, AstraZeneca, Tumura, Chugai, Wakayama Medical University, 
Hokkaido University, Mainichi Ga Hakken Inc., Boehringer Ingelheim, MSD Life Science Foundation, Taisho Pharmaceutical, and Scohia Pharma; grants from Mitsubishi Tanabe Pharma, Daiichi-Sankyo, AstraZeneca, Sumitomo Dainippon Pharma, Novartis, Novo Nordisk, Astellas, Sanofi, Terumo, Kyowa Hakko Kirin, Taisho Pharma, and Ono; endowments from Takeda, Kissei, Taisho Pharma, Sanofi, Daiichi-Sankyo, Mitsubishi Tanabe Pharma, Japan Tabacco Inc., Kyowa Hakko Kirin, Sumitomo Dainippon Pharma, Astellas, MSD, Ono, Sanwa Kagaku, Eli Lilly, Nippon Boehringer Ingelheim, Novartis, Novo Nordisk, Teijin Pharma, Shionogi, and The Japan China Medical Association. HW reports lecture fees from Takeda, Astellas, Sanofi, Mitsubishi Tanabe Pharma, Novo Nordisk, Kowa Pharmaceutical, AstraZeneca, Novartis, Nippon Boehringer Ingelheim, MSD, Sumitomo Dainippon Pharma, Eli Lilly, Sanwa Kagaku, Ono, Kissei, and Fujifilm Pharma; grants from Kowa Pharmaceutical, Novartis, Sanwa Kagaku, and Eli Lilly; endowments from Takeda, Astellas, Sanofi, Mitsubishi Tanabe Pharma, Novo Nordisk, AstraZeneca, Novartis, Nippon Boehringer Ingelheim, MSD, Sumitomo Dainippon Pharma, Kowa Pharmaceutical, Ono, Kyowa Hakko Kirin, Daiichi-Sankyo, Terumo, Pfizer, Mochida Pharmaceutical, Taisho Pharma, Johnson \& Johnson, Sanwa Kagaku, and Kowa. IS reports personal fees from Lotte, Kowa Life Science Foundation, Sunstar Foundation, MSD Life Science Foundation, Kanae Foundation, Senri Life Science Foundation, Fuji Foundation for Protein Research, Japan Cardiovascular Research Foundation, Japan Foundation for Applied Enzymology, Japan Society for the Promotion of Science, NPO Hormon Station, and Kawanishi medical association; lecture fees from Takeda, Japan Pharmacy Laboratory, Asia-Pacific Diabetes and Obesity Study Group, MSD, Astellas, AstraZeneca, Ono, Kyowa Hakko Kirin, Covidien Japan, Kowa Pharmaceutical, Sanofi, The Japan Society of Internal Medicine, Daiichi-Sankyo, Taisho Pharma, Sumitomo Dainippon Pharma, Mitsubishi Tanabe Pharma, Teijin Pharma, Eli Lilly, Nippon Boehringer Ingelheim, Japanese Society for Surgical Metabolism and Nutrition, Novartis, Novo Nordisk Mochida Pharmaceutical, Rohto Pharmaceutical, Sanwa Kagaku, and Astellas Amgen BioPharma; fees of speakers bureaus from Fuji Foundation for Protein Research; manuscript fees from lji Publishing, AstraZeneca, Ono, Kyowa Hakko Kirin, Sanwa Kagaku, Eli Lilly, Saishin Igaku-sha, Sentan Igaku-sha, and Medical Review-sha; consulting fees from Takeda, AstraZeneca, MSD, Ono, Kowa Pharmaceutical, Novartis, Novo Nordisk, Sanwa Kagaku, Nippon Boehringer Ingelheim, and Taisho Pharmaceutical; grants from AMED (Japan Agency for Medical Research and Development), National Center for Global Health and Medicine, Fukushima Medical University, Chiba University, Juntendo University, Kowa Pharmaceutical, Rohto Pharmaceutical, Otsuka Pharmaceutical, Sanwa Kagaku, and Immuno-Biological Laboratories; endowments from Takeda, MSD, Astellas, Nippon Becton Dickinson, Sumitomo Dainippon Pharma, Kyowa Hakko Kirin, Terumo, Mitsubishi Tanabe Pharma, Teijin Pharma, Shionogi, Kaken Pharmaceutical, Ono, Novartis, AstraZeneca, Sanofi, Eli Lilly, Daiichi-Sankyo, Kowa Pharmaceutical, Novo Nordisk, The Japan Diabetes Society, Midori Health Management Center, Japan Foundation for Applied Enzymology, MSD Life Science Foundation, Kowa Life Science Foundation, Suzuken Memorial Foundation, Japan Diabetes Foundation, Japan Heart Foundation, Gout Research Foundation, The Uehara Memorial Foundation, Takahashi Industrial and Economic Research Foundation, Soiken Holdings Inc., and Fukumoto Naika Clinic. RN reports lecture fees from Takeda, Sanofi, Medtronic Japan, Nippon Boehringer Ingelheim, Kissei, Novartis, Eli Lilly, Novo Nordisk, MSD, and Astellas; endowments from Japan Diabetes Foundation and Nippon Boehringer Ingelheim. HM reports lecture fees from Takeda, Novo Nordisk, Eli Lilly, Sanofi, Astellas, MSD, Mitsubishi Tanabe Pharma, Sumitomo Dainippon Pharma, Novartis, Ono, Kowa Pharmaceutical, Nippon Boehringer Ingelheim, AstraZeneca, Daiichi-Sankyo, Taisho Pharmaceutical, Teijin Pharma, and Sanwa Kagaku; grants from Astellas and Taisho Pharma; endowments from Takeda, Novo Nordisk, Eli Lilly, Nippon Boehringer Ingelheim, Daiichi-Sankyo, Ono, Sumitomo Dainippon Pharma, Astellas, MSD, Kowa Pharmaceutical, Taisho Pharmaceutical, Teijin Pharma, Sanwa Kagaku, and Kyowa Hakko Kirin. AA reports lecture fees from Takeda, Sanofi, Novo Nordisk, Eli Lilly, Ono, Nippon Boehringer Ingelheim, Kyowa Hakko Kirin, Sanwa Kagaku, Mitsubishi Tanabe Pharma, Astellas, AstraZeneca, MSD, Taisho Pharma, Daiichi-Sankyo, Novartis, Kowa Pharmaceutical, Bayer, Shionogi, Sumitomo Dainippon Pharma, and Fujifilm Pharma; consulting fees from Sanofi and Novo Nordisk; grants from Novo Nordisk and Taisho Pharma. HK reports lecture fees from Takeda, MSD, Novo Nordisk, Daiichi-Sankyo, Astellas, AstraZeneca, Ono, Kyowa Hakko Kirin, Kowa Pharmaceutical, Sanofi, Eli Lilly, Sanwa Kagaku, Taisho Pharma, Sumitomo Dainippon Pharma, Novartis, Mitsubishi Tanabe Pharma, Nippon Boehringer Ingelheim, Bayer, Medtronic Japan, and Alcon Japan; grants from Otsuka Pharmaceutical; endowments from MSD, Eli Lilly, Novo Nordisk, Takeda, Daiichi-Sankyo, Astellas, Sanofi, Novartis, Mitsubishi Tanabe Pharma, Ono, Kyowa Hakko Kirin, Nippon Boehringer Ingelheim, Taisho Pharma, Fujifilm Pharma, Kissei, Nippon Becton Dickinson. MH reports lecture fees from
Takeda, Astellas, MSD, Ono, Sumitomo Dainippon Pharma, Mitsubishi Tanabe Pharma, Daiichi-Sankyo, Nippon Boehringer Ingelheim, Kowa, Eli Lilly, Novo Nordisk, and Sanofi. AS reports lecture fees from MSD, Eli Lilly, Novo Nordisk, Sanofi, Mitsubishi Tanabe Pharma, Nippon Boehringer Ingelheim, Astellas, Ono, and Terumo; endowments from Takeda, MSD, Astellas, Novo Nordisk, Nippon Boehringer Ingelheim, Ono, and Daiichi-Sankyo. KN reports none. SF reports lecture fees from Takeda, Ono, AstraZeneca, Novo Nordisk, MSD, Daiichi-Sankyo, Kyowa Hakko Kirin, Eli Lilly, Kowa, Astellas, Sumitomo Dainippon Pharma, Asahi Kasei Pharma, and Nippon Boehringer Ingelheim; endowments from Takeda, Sanofi, Sumitomo Dainippon Pharma, MSD, Ono, Mitsubishi Tanabe Pharma, Eli Lilly, Novo Nordisk, Shionogi, Nippon Boehringer Ingelheim, Kyowa Hakko Kirin, Taisho Pharma, and Daiichi-Sankyo. MF reports none. KS reports lecture fees from Ono, Novartis, MSD, Astellas, Mitsubishi Tanabe Pharma, AstraZeneca, Sanofi Aventis, Novo Nordisk, Nippon Boehringer Ingelheim, Kyowa Hakko Kirin, Daiichi-Sankyo, and Eli Lilly; manuscript fees from Johnson \& Johnson and Kyowa Hakko Kirin; consulting fees from Daiichi-Sankyo; grants from Eli Lilly and Mitsubishi Tanabe Pharma; endowments from Takeda, Mitsubishi Tanabe Pharma, Kyowa Hakko Kirin, MSD, Astellas, Novartis, Ono, Sanofi Aventis, and Chugai. Y0 reports lecture fees from Takeda, MSD, Eli Lilly, Novartis, Sumitomo Dainippon Pharma, Sanofi, Daiichi-Sankyo, Sanwa Kagaku Kenkyusho, Novo Nordisk, Kissei, Boehringer Ingelheim, AstraZeneca, Astellas, Mitsubishi Tanabe Pharma, Kowa, Bayer, and Ono; grants from Mitsubishi Tanabe Pharma and Kowa. EA reports lecture fees from Takeda, Arkray, Astellas, AstraZeneca, Abbott Japan, Alcon, MSD, Ono, Kyowa Kikaku, Kyowa Hakko Kirin, Kissei, Kowa, Kowa Pharmaceutical, Sanofi, Sanwa Kagaku, Shionogi, Mitsubishi Tanabe Pharma, Daiichi-Sankyo, Taisho Pharmaceutical, Sumitomo Dainippon Pharma, Chugai, Terumo, Eli Lilly, Fujifilm Pharma, Nippon Boehringer Ingelheim, Novartis, Novo Nordisk, Bayer, Pfizer, Medical Review, Medtronic Japan, and MedScape; manuscript fees from Yodosha, Fuji Medical Publishing, and Life science Co; endowments from Takeda, Astellas, MSD, Ono, Kissei, Kyowa Hakko Kirin, Kowa, Sanofi, Shionogi, Taisho Pharmaceutical, Daiichi-Sankyo, Sumitomo Dainippon Pharma, Mitsubishi Tanabe Pharma, Terumo, Eli Lilly, Nippon Boehringer Ingelheim, Novartis, Novo Nordisk, and Mochida. TY reports lecture fees from Takeda, Daiichi-Sankyo, Mitsubishi Tanabe Pharma, AstraZeneca, Shionogi, and Sumitomo Dainippon Pharma. TK reports lecture fees from Takeda, Astellas, AstraZeneca, MSD, Ono, Eli Lilly, Nippon Boehringer Ingelheim, Novo Nordisk, Mitsubishi Tanabe Pharma, Sanofi, Novartis, Sanwa Kagaku Kenkyusho, Medtronic Japan, Sumitomo Dainippon Pharma, Kyowa Hakko Kirin, Abbott Japan, MedScape Education, Daiichi-Sankyo, and Taisho Pharmaceutical; manuscript fees from Takeda; grants from Takeda, DaiichiSankyo, AstraZeneca, Nippon Boehringer Ingelheim, and Eli Lilly; endowments from Takeda, Sumitomo Dainippon Pharma, Astellas, Taisho Pharma, Kyowa Hakko Kirin, Kissei, Novo Nordisk, Sanofi, Ono, Mitsubishi Tanabe Pharma, DaiichiSankyo, Kowa Pharmaceutical, MSD, Nippon Boehringer Ingelheim, Terumo, and Asahi Mutual Life Insurance Company.

Patient consent for publication Not required.

Provenance and peer review Not commissioned; externally peer reviewed.

Data availability statement Data are available on reasonable request. Details of the study protocol and statistical analysis plan will be available on reasonable request.

Supplemental material This content has been supplied by the author(s). It has not been vetted by BMJ Publishing Group Limited (BMJ) and may not have been peer-reviewed. Any opinions or recommendations discussed are solely those of the author(s) and are not endorsed by BMJ. BMJ disclaims all liability and responsibility arising from any reliance placed on the content. Where the content includes any translated material, BMJ does not warrant the accuracy and reliability of the translations (including but not limited to local regulations, clinical guidelines, terminology, drug names and drug dosages), and is not responsible for any error and/or omissions arising from translation and adaptation or otherwise.

Open access This is an open access article distributed in accordance with the Creative Commons Attribution Non Commercial (CC BY-NC 4.0) license, which permits others to distribute, remix, adapt, build upon this work non-commercially, and license their derivative works on different terms, provided the original work is properly cited, appropriate credit is given, any changes made indicated, and the use is non-commercial. See: http://creativecommons.org/licenses/by-nc/4.0/.

\section{ORCID iDs}

Kohjiro Ueki http://orcid.org/0000-0003-1523-8102

Nobuya Inagaki http://orcid.org/0000-0001-8261-2593

Hideaki Miyoshi http://orcid.org/0000-0002-5909-3243

Akira Shimada http://orcid.org/0000-0002-3954-4983 
Takashi Kadowaki http://orcid.org/0000-0002-5428-3582

\section{REFERENCES}

1 Cho NH, Shaw JE, Karuranga S, et al. IDF Diabetes Atlas: global estimates of diabetes prevalence for 2017 and projections for 2045. Diabetes Res Clin Pract 2018;138:271-81.

2 da Rocha Fernandes J, Ogurtsova K, Linnenkamp U, et al. IDF Diabetes Atlas estimates of 2014 global health expenditures on diabetes. Diabetes Res Clin Pract 2016;117:48-54.

3 Harding JL, Pavkov ME, Magliano DJ, et al. Global trends in diabetes complications: a review of current evidence. Diabetologia 2019;62:3-16.

4 American Diabetes Association. 8. Pharmacologic approaches to glycemic treatment: standards of medical care in diabetes -2018 . Diabetes Care 2018;41:S73-85.

5 Umezawa S, Kubota A, Maeda H, et al. Two-year assessment of the efficacy and safety of sitagliptin in elderly patients with type 2 diabetes: post hoc analysis of the ASSET-K study. BMC Endocr Disord 2015;15:34.

6 Del Prato S, Fleck P, Wilson C, et al. Comparison of alogliptin and glipizide for composite endpoint of glycated haemoglobin reduction, no hypoglycaemia and no weight gain in type 2 diabetes mellitus. Diabetes Obes Metab 2016;18:623-7.

7 Scirica BM, Bhatt DL, Braunwald E, et al. Saxagliptin and cardiovascular outcomes in patients with type 2 diabetes mellitus. $N$ Engl J Med 2013;369:1317-26.

8 White WB, Cannon CP, Heller SR, et al. Alogliptin after acute coronary syndrome in patients with type 2 diabetes. N Engl J Med 2013;369:1327-35.

9 Green JB, Bethel MA, Armstrong PW, et al. Effect of sitagliptin on cardiovascular outcomes in type 2 diabetes. $N$ Engl J Med 2015;373:232-42.

10 Rosenstock J, Perkovic V, Johansen OE, et al. Effect of linagliptin vs placebo on major cardiovascular events in adults with type 2 diabetes and high cardiovascular and renal risk. JAMA 2019;321:69-79.

11 Stoian AP, Sachinidis A, Stoica RA, et al. The efficacy and safety of dipeptidyl peptidase-4 inhibitors compared to other oral glucose-lowering medications in the treatment of type 2 diabetes. Metabolism 2020;109:154295.

12 Li L, Li S, Deng K, et al. Dipeptidyl peptidase-4 inhibitors and risk of heart failure in type 2 diabetes: systematic review and meta-analysis of randomised and observational studies. BMJ 2016;352:i610.

13 Zannad F, Cannon CP, Cushman WC, et al. Heart failure and mortality outcomes in patients with type 2 diabetes taking alogliptin versus placebo in EXAMINE: a multicentre, randomised, doubleblind trial. Lancet 2015;385:2067-76.

14 Gitt AK, Bramlage P, Binz C, et al. Prognostic implications of DPP-4 inhibitor vs. sulfonylurea use on top of metformin in a real world setting - results of the 1 year follow-up of the prospective DiaRegis registry. Int J Clin Pract 2013;67:1005-14.

15 Ou S-M, Shih C-J, Chao P-W, et al. Effects on clinical outcomes of adding dipeptidyl peptidase-4 inhibitors versus sulfonylureas to metformin therapy in patients with type 2 diabetes mellitus. Ann Intern Med 2015;163:663-72.

16 Inagaki N, Ueki K, Tanizawa Y, et al. Protocol for a large-scale prospective observational study with alogliptin in patients with type 2 diabetes: J-BRAND Registry. BMJ Open 2014;4:e004760.
17 Tajima N, Noda M, Origasa $\mathrm{H}$, et al. Evidence-based practice guideline for the treatment for diabetes in Japan 2013. Diabetol Int 2015;6:151-87.

18 Gooßen K, Gräber S. Longer term safety of dipeptidyl peptidase-4 inhibitors in patients with type 2 diabetes mellitus: systematic review and meta-analysis. Diabetes Obes Metab 2012;14:1061-72.

19 Elashoff M, Matveyenko AV, Gier B, et al. Pancreatitis, pancreatic, and thyroid cancer with glucagon-like peptide-1-based therapies. Gastroenterology 2011;141:150-6.

20 Chijiwa C, Takeoka S, Kamata M, et al. Decrease in eosinophils infiltrating into the skin of patients with dipeptidyl peptidase-4 inhibitor-related bullous pemphigoid. J Dermatol 2018;45:596-9.

21 Karagiannis T, Paschos P, Paletas K, et al. Dipeptidyl peptidase-4 inhibitors for treatment of type 2 diabetes mellitus in the clinical setting: systematic review and meta-analysis. BMJ 2012;344:e1369.

22 Imatoh T, Nishi T, Yasui M, et al. Association between dipeptidyl peptidase-4 inhibitors and urinary tract infection in elderly patients: a retrospective cohort study. Pharmacoepidemiol Drug Saf 2018;27:931-9.

23 Kridin K, Bergman R. Association of bullous pemphigoid with dipeptidyl-peptidase 4 inhibitors in patients with diabetes: estimating the risk of the new agents and characterizing the patients. JAMA Dermatol 2018;154:1152-8.

24 Kridin K, Cohen AD. Dipeptidyl-peptidase IV inhibitor-associated bullous pemphigoid: a systematic review and meta-analysis. J Am Acad Dermatol 2018;S0190-9622:32660-4.

25 Butler AE, Campbell-Thompson M, Gurlo T, et al. Marked expansion of exocrine and endocrine pancreas with incretin therapy in humans with increased exocrine pancreas dysplasia and the potential for glucagon-producing neuroendocrine tumors. Diabetes 2013;62:2595-604.

26 Ujiie $\mathrm{H}$, Muramatsu K, Mushiroda T, et al. HLA-DQB1*03:01 as a biomarker for genetic susceptibility to bullous pemphigoid induced by DPP-4 inhibitors. Journal of Investigative Dermatology 2018;138:1201-4.

27 Effect of intensive blood-glucose control with metformin on complications in overweight patients with type 2 diabetes (UKPDS 34). Lancet 1998;352:854-65.

28 Zelniker TA, Wiviott SD, Raz I, et al. SGLT2 inhibitors for primary and secondary prevention of cardiovascular and renal outcomes in type 2 diabetes: a systematic review and meta-analysis of cardiovascular outcome trials. The Lancet 2019;393:31-9.

29 U.K. prospective diabetes study 16. Overview of 6 years' therapy of type II diabetes: a progressive disease. U.K. Prospective Diabetes Study Group. Diabetes 1995;44:1249-58.

30 Kahn SE, Haffner SM, Heise MA, et al. Glycemic durability of rosiglitazone, metformin, or glyburide monotherapy. N Engl J Med Overseas Ed 2006;355:2427-43.

$31 \mathrm{Kim}$ YG, Hahn S, Oh TJ, et al. Differences in the glucose-lowering efficacy of dipeptidyl peptidase-4 inhibitors between Asians and non-Asians: a systematic review and meta-analysis. Diabetologia 2013;56:696-708.

32 Ogurtsova K, da Rocha Fernandes JD, Huang Y, et al. IDF Diabetes Atlas: global estimates for the prevalence of diabetes for 2015 and 2040. Diabetes Res Clin Pract 2017;128:40-50.

33 Namba M, Iwakura T, Nishimura R, et al. The current status of treatment-related severe hypoglycemia in Japanese patients with diabetes mellitus: a report from the committee on a survey of severe hypoglycemia in the Japan Diabetes Society. Diabetol Int 2018;9:84-99. 